\title{
(Anti-)Chiral Supervariable Approach to Nilpotent and Absolutely Anticommuting Conserved Charges of Reparameterization Invariant Theories: A Couple of Relativistic Toy Models as Examples
}

\author{
S. $\operatorname{Kumar}^{(a)}$, B. Chauhan ${ }^{(a)}$, R. P. Malik ${ }^{(a, b)}$ \\ (a) Physics Department, Center of Advance Studies, Institute of Science, \\ Banaras Hindu University, Varanasi - 221 005, (U.P.), India \\ (b) DST Center for Interdisciplinary Mathematical Sciences, \\ Institute of Science, Banaras Hindu University, Varanasi - 221 005, India \\ e-mails: sunil.bhu93@gmail.com; bchauhan501@gmail.com; rpmalik1995@gmail.com
}

\begin{abstract}
We exploit the potential and power of the Becchi-Rouet-Stora-Tyutin (BRST) and anti-BRST invariant restrictions on the (anti-)chiral supervariables to derive the proper nilpotent (anti-)BRST symmetries for the reparameterization invariant one $(0+1)$ dimensional (1D) toy models of a free relativistic particle as well as a free spinning (i.e. supersymmetric) relativistic particle within the framework of (anti-)chiral supervariable approach to BRST formalism. Despite the (anti-)chiral super expansions of the (anti-)chiral supervariables, we observe that the (anti-)BRST charges, for the above toy models, turn out to be absolutely anticommuting in nature. This is one of the novel observations of our present endeavor. For this proof, we utilize the beauty and strength of Curci-Ferrari (CF)-type restriction in the context of a spinning relativistic particle but no such restriction is required in the case of a free scalar relativistic particle. We have also captured the nilpotency property of the conserved charges as well as the (anti-)BRST invariance of the appropriate Lagrangian(s) of our present toy models within the framework of (anti-)chiral supervariable approach.
\end{abstract}

PACS numbers: 11.15.-q; 12.20.-m; 11.30.Ph; 02.20.+b

Keywords: (Anti-)chiral supervariable approach; a free scalar relativistic particle; a free massless spinning relativistic particle; off-shell nilpotent (anti-)BRST symmetries; nilpotent (anti-)BRST charges; absolute anticommutativity of the (anti-)BRST charges; chiral and anti-chiral super expansion(s); (anti-)BRST invariant restrictions; Curci-Ferrari type restriction; coupled Lagrangians 


\section{Introduction}

The usual superfield approach (USFA) to Becchi-Rouet-Stora-Tyutin (BRST) formalism [1-8] exploits the idea of horizontality condition (HC) where the concepts from differential geometry play a decisive role. This approach enables us to derive the (anti-)BRST symmetries associated with the $p$-form $(p=1,2,3, \ldots)$ gauge field and associated (anti-)ghost fields of a given $p$-form gauge theory within the framework of BRST formalism. In addition, it also leads to the derivation of Curci-Ferrari $(\mathrm{CF})$ condition [9] which is the hallmark $[10,11]$ of a given quantum gauge theory within the framework of BRST formalism. Moreover, the USFA (with the help of the geometrical $\mathrm{HC}$ ) also sheds light on the geometrical meaning of the nilpotency and absolute anticommutativity properties of the BRST and anti-BRST symmetries and corresponding conserved charges (which are primarily mathematical in nature without the knowledge of USFA to BRST formalism). These observations are true for a given free $p$-form gauge theory (in any arbitrary dimension of spacetime) where there is no interaction between the gauge field and matter field(s).

The above USFA has been systematically generalized in our earlier works [12-16] where we have exploited the theoretical strength of gauge invariant restrictions (along and consistent with HC) so as to derive the (anti-)BRST symmetries associated with the matter, gauge and (anti-)ghost fields together. This generalized version of superfield approach to BRST formalism has been christened as the augmented superfield approach (ASFA) to BRST formalism in our earlier works [12-16]. In our present endeavor, we shall exploit the theoretical potential and power of ASFA* to BRST formalism to capture the nilpotency and absolute anticommutativity properties of the (anti-)BRST charges for the reparameterization invariant theories of a 1D free massive scalar and a massless spinning relativistic particles. The clinching proof of the absolute anticommutativity property of the conserved and nilpotent (anti-)BRST charges is a novel observation in our present endeavor.

In all the above superfield approaches [1-8, 12-16], we have taken super expansions of the superfields along all possible Grassmannian directions of the appropriately chosen $(\mathrm{D}, 2)$-dimensional supermanifold on which a given $\mathrm{D}$-dimensional $p$-form gauge theory is generalized. In our recent set of papers [17-22], we have taken the help of (anti-)chiral supervariables/superfields to derive the (anti-)BRST as well as (anti-)co-BRST symmetry transformations for the 1D toy model of a rigid rotor [17, 18], 2D model of a self-dual bosonic field theory [19] and 4D model of an Abelian 2-form gauge theory [20]. To derive the above symmetries, we have utilized the idea of symmetry invariance where we have invoked the (anti-)BRST and (anti-)co-BRST invariant restrictions on the supervariables/superfields. We have also applied the above idea in the context of interacting Abelian and non-Abelian gauge theories where there are interactions between the gauge and matter fields [21, 22]. One of the novel observations of our earlier endeavors [17-22] has been the proof of absolute anticommutativity of the (anti-)BRST (and (anti-)co-BRST) charges despite the fact that we have considered only the (anti-)chiral super expansions of the supervariables/superfields. This observation has been established only in the context of gauge theories within the

${ }^{*}$ To be precise, we shall utilize the augmented version of the supervariable approach to BRST formalism where we shall consider only the (anti-)chiral super expansions of the supervariables. We christen our approach as " the supervariable approach" because we are dealing with "variables" and not the "fields" in our present endeavor (where we are concerned with only the 1D toy models of relativistic particles). 
framework of (anti-)chiral superfield approach to BRST formalism.

The central theme of our present investigation is to exploit the simplicity and beauty of the augmented version of (anti-)chiral supervariable approach [(A)CSVA] to BRST formalism in the context of reparameterization invariant theories of $1 \mathrm{D}$ toy models of a free scalar relativistic particle as well as a spinning relativistic particle to establish the absolute anticommutativity property of the (anti-)BRST charges. In the process, we also capture the nilpotency of the (anti-)BRST charges and the (anti-)BRST invariance of the appropriate Lagrangian(s) of the above models. The present reparameterization invariant theories are important because these are precursors to the (super)string and supergravity theories where the idea of reparameterization invariance plays a key role. It is worthwhile to point out that the gauge and reparameterization symmetries of the present toy models have been shown to be equivalent when the free motion $\left(\dot{p}_{\mu}=0\right)$ of the free relativistic particles and the specific relationship between the gauge and reparameterization transformation parameters are taken into account together (see. e.g. [24] for details).

We would like to lay emphasis that, in the proof of absolute anticommutativity of the (anti-)BRST charges, we have utilized the beauty and strength of CF-type restriction which has been systematically and elegantly derived in our earlier work in the case of a 1D toy model of a spinning relativistic particle [23]. However, there is no CF-type restriction in the context of a free massive scalar relativistic particle. As a consequence, for the proof of the absolute anticommutativity of the conserved (anti-)BRST charges (in the case of this 1D toy model), there is no requirement of invoking any kind of restriction from outside. Our present endeavor completes our program of proving the nilpotency and absolute anticommutativity of the (anti-)BRST charges within the framework of (A)CSVA/(A)CSFA to gauge invariant theories and reparameterization invariant theories (where only the (anti-) chiral super expansions of the supervariables/superfields are utilized). We re-emphasize that, for the 1D toy models under consideration in our present endeavor, the gauge and reparameterization symmetries are intertwined together in a beautiful manner and they are found to be equivalent on-shell under very specific condition where the transformation parameters of these symmetry transformations are related with each-other in a particular fashion (see, e.g. [24] for details).

We enumerate a few novel features that are associated with the discussion of a free spinning relativistic particle (against the backdrop of our discussions connected with a free scalar relativistic particle) within the framework of (A)CSVA to BRST formalism. The conserved (anti-)BRST charges (cf. Eq. (4) below), derived directly from the application of the Noether theorem, are found to be off-shell nilpotent in the case of a free scalar relativistic particle. However, the same charges $Q_{(a) b}^{(1)}$ (cf. Eq. (13) below), derived directly by exploiting the virtues of Noether's theorem, turn out to be on-shell nilpotent (despite the fact that we have used the (anti-)BRST symmetries (cf. Eqs. (9), (10) below) which are off-shell nilpotent). These charges become off-shell nilpotent only when we use the appropriate EOMs (cf. Eqs. (14), (15) below) to recast them in a different form $Q_{(a) b}^{(2)}$

\footnotetext{
${ }^{\dagger}$ We call our approach as the supervariable approach to BRST formalism because, in the limiting case (when the Grassmannian coordinates are set equal to zero), we obtain a variable from the super expansion(s) of the supervariable(s). This observation should be contrasted with the superfield approach to BRST formalism where we obtain a field, in the above limit, from the super expansion(s) of the superfield(s).
} 
(cf. Eqs. (13), (17) below). In exactly similar fashion, the (anti-)BRST charges (cf. Eq. (4) below) are absolutely anticommuting without any use of EOMs and/or any kind of outside restriction(s) (e.g. CF-type condition(s)). This is not the case with the (anti-)BRST charges associated with the spinning relativistic particle under consideration. In the proof of absolute anticommutativity property of the (anti-)BRST charges for a spinning relativistic particle, we have to first recast the expressions for the off-shell nilpotent charges (cf. Eq. (17) below) in a different form (cf. Eq. (63) below) by utilizing the appropriate EOMs as well as the CF-type restriction and, then only, we have to exploit the idea of continuous symmetries and their generators to prove the absolute anticommutativity property of the (anti-)BRST charges (cf. Eqs. (66), (70) below).

Our present investigation is essential and interesting on the following grounds. First, we have applied, so far, our (A)CSVA/(A)CSFA to only 1D, 2D and 4D models of gauge theories and derived the (anti-)BRST as well as (anti-)co-BRST symmetries. Thus, it is of immense importance for us to apply it to the models of reparametrization invariant theories (which are the precursors to the (super)string and supergravity theories). Second, the results of our present investigation establish that the absolute anticommutativity of the (anti-)BRST charges is universal in the case of gauge- and reparameterization invariant theories despite the fact that we have taken into account only the (anti-)chiral super expansions for the supervariables/superfields within the framework of (A)CSVA/(A)CSFA to BRST formalism. Finally, the reparameterization invariant models (under consideration) are interesting and important in their own right as they also represent the ordinary as well as the supersymmetric prototype toy models of theoretical (and mathematical) interests in one $(0+1)$-dimension of spacetime.

Our present paper is organized as follows. First of all, to fix the notations and convention, we discuss concisely, in Sec. 2, the bare essentials of a free scalar relativistic and a free spinning relativistic particles within the framework of (anti-) BRST invariant Lagrangians where we elaborate on the nilpotent (anti-)BRST symmetries and derive their corresponding conserved charges. Our Sec. 3 is devoted to the derivation of (anti-)BRST symmetry transformations for a free scalar relativistic particle within the framework of (A)CSVA to BRST formalism where we invoke the (anti-)BRST invariant restrictions on the (anti-)chiral supervariables. In Sec. 4, we derive the nilpotent (anti-)BRST symmetry transformations for a spinning relativistic particle by exploiting the beauty and strength of the (anti-)BRST invariant restrictions on the (anti-)chiral supervariables (that are fermionic, bosonic and their appropriate combinations). Our Sec. 5 deals with the proof of nilpotency and absolute anticommutativity of the (anti-)BRST charges for both of our models within the framework of (A)CSVA to BRST formalism. Finally, we summarize our key results, comment on some crucial issues related to our $1 \mathrm{D}$ reparameterization invariant toy models and point out a few future directions for further investigations in our last section (i.e. Sec. 6).

In our Appendices $\mathrm{A}, \mathrm{B}$ and $\mathrm{C}$, we elaborate on a few theoretical computations that have either been incorporated into the main body of our text or they supplement our key results. Particularly, in our Appendix C, we capture the (anti-)BRST invariance of the

\footnotetext{
${ }^{\ddagger}$ It can be checked that the infinitesimal version of reparameterization transformation leaves the action integrals, corresponding to the appropriate Lagrangian(s) of our 1D toy models, invariant (see, e.g. Refs. [23-25] for details).
} 
Lagrangian(s) of the two relativistic toy models (existing in one (0+1)-dimensional spacetime) that have been considered in our present endeavor.

Convention and Notations: Through out the whole body of our text, we take the convention of left-derivative w.r.t. all the fermionic variables (e.g. $\left.c, \bar{c}, \chi, \psi_{\mu}, \gamma\right)$ for all the appropriate computations that are connected with the 1D toy models of our present endeavor. We also use the notations $s_{(a) b}$ and $Q_{(a) b}$ for the off-shell nilpotent (anti-)BRST symmetry transformations and corresponding off-shell nilpotent (anti-)BRST charges for both the relativistic toy models which are examples of the reparameterization invariant theories.

\section{Preliminary: Off-Shell Nilpotent BRST and Anti- BRST Symmetries in Lagrangian Formulation}

We begin with the following (anti-)BRST invariant first-order Lagrangian for a free scalar relativistic particle of rest mass $m$ (see, e.g. [24, 25]):

$$
L_{b}=p_{\mu} \dot{x}^{\mu}-\frac{1}{2} e\left(p^{2}-m^{2}\right)+b \dot{e}+\frac{1}{2} b^{2}-i \dot{\bar{c}} \dot{c}
$$

where the canonically conjugate target space variables $\left(x_{\mu}(\tau), p^{\mu}(\tau)\right)$ are the D-dimensional (i.e. $\mu, \nu, \lambda, \ldots=0,1,2, \ldots, D-1)$ coordinates and momenta, $e(\tau)$ is an einbein variable, $b(\tau)$ is the Nakanishi-Lautrup auxiliary variable and (anti-)ghost variables $(\bar{c}(\tau)) c(\tau)$ are needed for the unitarity in the theory. The above Lagrangian describes the free motion $\left(\dot{p}_{\mu}=0\right)$ of a scalar relativistic particle on a world-line parametrized by $\tau$ and this world-line is embedded in the D-dimensional target space. Thus, all the variables of this theory are function of $\tau$ and, therefore, we have $\dot{p}_{\mu}=\frac{d p_{\mu}}{d \tau}$. It is evident that $\Pi_{e} \approx 0$ and $p^{2}-m^{2} \approx 0$ are the first-class constraints on the theory in the terminology of Dirac's prescription for the classification scheme of constraints $[26,27]$. Here $\Pi_{e}$ is the canonical conjugate momentum w.r.t. the einbein variable $e(\tau)$. The above constraints generate the local gauge transformations which can be generalized to the (anti-)BRST symmetry transformations $s_{(a) b}$ as (see, e.g. [24, 25] for details):

$$
\begin{aligned}
s_{a b} x_{\mu} & =\bar{c} p_{\mu}, s_{a b} \bar{c}=0, s_{a b} p_{\mu}=0, s_{a b} c=-i b, s_{a b} b=0, s_{a b} e=\dot{\bar{c}}, \\
s_{b} x_{\mu} & =c p_{\mu}, s_{b} c=0, s_{b} p_{\mu}=0, s_{b} \bar{c}=i b, s_{b} b=0, s_{b} e=\dot{c} .
\end{aligned}
$$

It is straightforward to check that the above transformations are off-shell nilpotent of order two (i.e. $\left.s_{(a) b}^{2}=0\right)$ and absolutely anticommuting (i.e. $s_{b} s_{a b}+s_{a b} s_{b}=0$ ) in nature. We note that, under the above (anti-)BRST symmetry transformations (2), the Lagrangian (1) transforms to the total derivatives w.r.t. the evolution parameter $\tau$, as

$$
s_{b} L_{b}=\frac{d}{d \tau}\left[\frac{1}{2} c\left(p^{2}+m^{2}\right)+b \dot{c}\right], \quad s_{a b} L_{b}=\frac{d}{d \tau}\left[\frac{1}{2} \bar{c}\left(p^{2}+m^{2}\right)+b \dot{\bar{c}}\right],
$$

thereby rendering the action integral $S=\int d \tau L_{b}$ (corresponding to the starting Lagrangian $L_{b}$ ) invariant for the physically well-defined variables that vanish-off at $\tau= \pm \infty$ due to Gauss's divergence theorem. 
According to Noether's theorem, the above continuous symmetries lead to the derivation of conserved $\left(\dot{Q}_{(a) b}=0\right)$ (anti-)BRST charges $Q_{(a) b}$ as

$$
Q_{b}=\frac{c}{2}\left(p^{2}-m^{2}\right)+b \dot{c} \equiv b \dot{c}-\dot{b} c, \quad Q_{a b}=\frac{\bar{c}}{2}\left(p^{2}-m^{2}\right)+b \dot{\bar{c}} \equiv b \dot{\bar{c}}-\dot{b} \bar{c},
$$

where we have used the equation of motion w.r.t. $e$ which leads to: $\dot{b}=-\frac{1}{2}\left(p^{2}-m^{2}\right)$ in the final expressions for $Q_{(a) b}$. The conserved charges $Q_{(a) b}$ are the generators of the (anti-) BRST symmetry transformations in (2). The nilpotency and absolute anticommutativity of the (anti-)BRST charges (cf. Eq. (4)) can be proven in a straightforward fashion as follows

$$
\begin{array}{ll}
s_{b} Q_{b}=-i\left\{Q_{b}, Q_{b}\right\}=0, & s_{a b} Q_{b}=-i\left\{Q_{b}, Q_{a b}\right\}=0, \\
s_{a b} Q_{a b}=-i\left\{Q_{a b}, Q_{a b}\right\}=0, & s_{b} Q_{a b}=-i\left\{Q_{a b}, Q_{b}\right\}=0,
\end{array}
$$

where we have used the property of $Q_{(a) b}$ as the generators for all the continuous symmetry transformations $s_{(a) b}$. We have also applied the symmetry transformations (2) directly on $Q_{(a) b}$ to prove that $s_{b} Q_{b}=0, s_{a b} Q_{a b}=0, s_{b} Q_{a b}=i(b \dot{b}-\dot{b} b)=0$ and $s_{a b} Q_{b}=-i(b \dot{b}-\dot{b} b)=$ 0 .

Now we dwell a bit on a massless spinning relativistic particle which is described by the following (anti-)BRST invariant coupled (but equivalent) Lagrangians (see, e.g. [23])

$$
\begin{aligned}
L_{B} & =L_{0}+b \dot{e}+b(b+2 \beta \bar{\beta})-i \dot{\bar{c}}(\dot{c}+2 \beta \chi)+2 i \bar{\beta} \dot{c} \chi \\
& -2 e(\gamma \chi+\bar{\beta} \dot{\beta})+2 \beta \gamma \bar{c}+\bar{\beta}^{2} \beta^{2}+2 \bar{\beta} c \gamma, \\
L_{\bar{B}} & =L_{0}-\bar{b} \dot{e}+\bar{b}(\bar{b}+2 \bar{\beta} \beta)-i \dot{\bar{c}}(\dot{c}+2 \beta \chi)+2 i \bar{\beta} \dot{c} \chi \\
& -2 e(\gamma \chi-\beta \overline{\bar{\beta}})+2 \beta \gamma \bar{c}+\bar{\beta}^{2} \beta^{2}+2 \bar{\beta} c \gamma,
\end{aligned}
$$

where $L_{0}$ is the first-order Lagrangian for the 1D toy model of a free massless spinning relativistic particle as follows (see, e.g. [24] for details):

$$
L_{0}=p_{\mu} \dot{x}^{\mu}-\frac{e}{2} p^{2}+\frac{i}{2} \psi_{\mu} \dot{\psi}^{\mu}+i \chi\left(p_{\mu} \psi^{\mu}\right)
$$

In the above, the constraints $p^{2} \approx 0$ and $p_{\mu} \psi^{\mu} \approx 0$ are the first-class in the terminology of Dirac's prescription for the classification of constraints and these have been incorporated into the above Lagrangian through the Lagrange multiplier variables $e(\tau)$ and $\chi(\tau)$. The latter variables are the analogs of the vierbein and Rarita-Schwinger (i.e. gravitino) fields of the $4 \mathrm{D}$ supergravity theory. In our present discussion, these variables $e(\tau)$ and $\chi(\tau)$ are also the analogs of gauge fields of the $4 \mathrm{D}$ gauge theory. The fermionic variables $\psi_{\mu}$ are the superpartners of $x_{\mu}$ and they satisfy: $\left(\psi_{\mu}\right)^{2}=0, \psi_{\mu} \psi_{\nu}+\psi_{\nu} \psi_{\mu}=0, \chi \psi_{\mu}+\psi_{\mu} \chi=0$ because $\chi(\tau)$ is also fermionic in nature and it is the superpartner of the einbein variable $e(\tau)$. We point out that the super world-line, traced out by the motion of the spinning massless relativistic particle, is parameterized by $\tau$ and it is embedded in the D-dimensional target space supermanifold where $\mu, \nu, \lambda, \ldots=0,1,2, \ldots D-1$. It is straightforward to conclude that all the variables of our present toy model are function of the evolution parameter $\tau$ and $\dot{x}_{\mu}=\frac{d x_{\mu}}{d \tau}, \dot{\psi}_{\mu}=\frac{d \psi_{\mu}}{d \tau}$. 
We observe that both the above Lagrangians are equivalent because both of them respect the (anti-)BRST symmetries provided we use the Curci-Ferrari (CF) type restrictions: $b+\bar{b}+2 \bar{\beta} \beta=0$ which emerges from the Euler-Lagrange equations of motion derived from the above Lagrangians, namely;

$$
b=-\frac{1}{2} \dot{e}-\bar{\beta} \beta, \quad \bar{b}=\frac{1}{2} \dot{e}-\bar{\beta} \beta \Longrightarrow b+\bar{b}+2 \bar{\beta} \beta=0 .
$$

We shall consider our BRST and anti-BRST symmetries only on a hyper world-line (embedded in the D-dimensional target space) where the above CF-type restriction is satisfied because the absolute anticommutativity (i.e. $\left\{s_{b}, s_{a b}\right\}=0$ ) of the off-shell nilpotent (i.e. fermionic; $\left.s_{(a) b}^{2}=0\right)$ (anti-)BRST symmetries $s_{(a) b}$ is also satisfied only on this hyper worldline. For instance, it can be checked that $\left\{s_{b}, s_{a b}\right\} e=0$ and $\left\{s_{b}, s_{a b}\right\} x_{\mu}=0$ only when we use the CF-type restriction: $b+\bar{b}+2 \beta \bar{\beta}=0$ in their proof of the absolute anticommutativity properties (i.e. $s_{b} s_{a b}+s_{a b} s_{b}=0$ ).

In the above coupled Lagrangians, the auxiliary variables $(b, \bar{b})$ are the NakanishiLautrup variables, $(\bar{c}) c$ are the fermionic $\left(c^{2}=\bar{c}^{2}=0, c \bar{c}+\bar{c} c=0\right)$ (anti-)ghost variables, $(\bar{\beta}) \beta$ are the bosonic (anti-)ghost variables, $(e, \chi)$ are the gauge and super-gauge variables and $\gamma$ is a fermionic $\left(\gamma^{2}=0\right)$ auxiliary variable. It can be checked that, under the following off-shell nilpotent $\left(s_{(a) b}^{2}=0\right)$ (anti-)BRST symmetry transformations $s_{(a) b}$

$$
\begin{aligned}
& s_{a b} x_{\mu}=\bar{c} p_{\mu}+\bar{\beta} \psi_{\mu}, \quad s_{a b} e=\dot{\bar{c}}+2 \bar{\beta} \chi, \quad s_{a b} \psi_{\mu}=i \bar{\beta} p_{\mu}, \\
& s_{a b} \bar{c}=-i \bar{\beta}^{2}, \quad s_{a b} c=i \bar{b}, \quad s_{a b} \bar{\beta}=0, \quad s_{a b} \beta=-i \gamma, \quad s_{a b} p_{\mu}=0, \\
& s_{a b} \gamma=0, \quad s_{a b} \chi=i \overline{\bar{\beta}}, \quad s_{a b} b=2 i \bar{\beta} \gamma, \\
& s_{b} x_{\mu}=c p_{\mu}+\beta \psi_{\mu}, \quad s_{b} e=\dot{\bar{b}}+2 \beta \chi, \quad s_{b} \psi_{\mu}=i \beta p_{\mu}, \\
& s_{b} c=-i \beta^{2}, \quad s_{b} \bar{c}=i b, \quad s_{b} \beta=0, \quad s_{b} \bar{\beta}=i \gamma, \quad s_{b} p_{\mu}=0, \\
& s_{b} \gamma=0, \quad s_{b} b=0, \quad s_{b} \chi=i \dot{\beta}, \quad s_{b} \bar{b}=-2 i \beta \gamma,
\end{aligned}
$$

the Lagrangians $L_{B}$ and $L_{\bar{B}}$ transform to total derivatives w.r.t. the evolution parameter $\tau$ (which characterizes the super world-line) as:

$$
\begin{gathered}
s_{b} L_{B}=\frac{d}{d \tau}\left[\frac{1}{2} c p^{2}+\frac{\beta}{2}(p \cdot \psi)+b(\dot{c}+2 \beta \chi)\right], \\
s_{a b} L_{\bar{B}}=\frac{d}{d \tau}\left[\frac{1}{2} \bar{c} p^{2}+\frac{\bar{\beta}}{2}(p \cdot \psi)-\bar{b}(\dot{\bar{c}}+2 \bar{\beta} \chi)\right] .
\end{gathered}
$$

As a consequence of the above explicit transformations, it is evident that the corresponding action integrals (i.e. $S_{1}=\int d \tau L_{B}$ and $S_{2}=\int d \tau L_{\bar{B}}$ ) would remain invariant under the BRST and anti-BRST symmetry transformations for the physically well-defined variables that vanish-off at $\tau= \pm \infty$.

$\S$ We have considered the supersymmetrization of the horizontality condition in our earlier work [23] on a massless as well as a massive spinning relativistic particle and have derived this specific CF-type restriction from the superfield/supervariable approach to our present reparameterization invariant theory. 
Invariance of the action, under the continuous symmetry transformations, leads to the derivation of the Noether conserved currents and corresponding conserved charges for our $1 \mathrm{D}$ system. We have the following equivalent expressions for the conserved (anti-)BRST charges (taking into account the EOM: $\left.\beta \dot{\bar{c}}=-\frac{1}{2}(p \cdot \psi)+i e \gamma+\bar{\beta} \dot{c}\right)$, namely;

$$
\begin{aligned}
Q_{a b}^{(1)} & =\frac{1}{2} \bar{c} p^{2}-\bar{b} \dot{\bar{c}}+\bar{\beta}(p \cdot \psi)-\bar{\beta}^{2} \dot{c}-2 \beta \bar{\beta}^{2} \chi-2 \bar{b} \bar{\beta} \chi \\
Q_{a b}^{(2)} & \equiv \frac{1}{2} \bar{c} p^{2}-\bar{b} \dot{\bar{c}}-\bar{\beta} \beta \dot{\bar{c}}+i \bar{\beta} e \gamma+\frac{1}{2} \bar{\beta}(p \cdot \psi)-2 \bar{\beta}^{2} \beta \chi-2 \bar{b} \bar{\beta} \chi, \\
Q_{b}^{(1)} & =\frac{1}{2} c p^{2}+b \dot{c}+\beta(p \cdot \psi)+\beta^{2} \dot{\bar{c}}+2 \beta^{2} \bar{\beta} \chi+2 b \beta \chi \\
Q_{b}^{(2)} & \equiv \frac{1}{2} c p^{2}+b \dot{c}+\beta \bar{\beta} \dot{c}+i \beta e \gamma+\frac{1}{2} \beta(p \cdot \psi)+2 \beta^{2} \bar{\beta} \chi+2 b \beta \chi,
\end{aligned}
$$

where, in expressing the equivalent forms of the charges, we have used the following equations of motion (EOMs) derived from the Lagrangian $L_{B}$, namely;

$$
\begin{aligned}
& \dot{p}_{\mu}=0, \quad \dot{x}_{\mu}=e p_{\mu}-i \chi \psi_{\mu}, \quad \dot{\psi}_{\mu}=\chi p_{\mu}, \quad \dot{e}+2 \beta \bar{\beta}+2 b=0, \\
& \dot{b}=-\frac{p^{2}}{2}-2 \gamma \chi-2 \bar{\beta} \dot{\beta}, \quad e \dot{\bar{\beta}}+\dot{e} \bar{\beta}+b \bar{\beta}-i \dot{\bar{c}} \chi+\gamma \bar{c}+\bar{\beta}^{2} \beta=0, \\
& \beta b+i \dot{c} \chi-e \dot{\beta}+\bar{\beta} \beta^{2}+c \gamma=0, \quad 2 \beta \dot{\bar{c}}-2 \bar{\beta} \dot{c}-2 i e \gamma+(p \cdot \psi)=0, \\
& \beta \bar{c}-\bar{\beta} c-e \chi=0, \quad \ddot{\bar{c}}+2 \dot{\bar{\beta}} \chi+2 \bar{\beta} \dot{\chi}+2 i \bar{\beta} \gamma=0, \\
& \ddot{c}+2 i \beta \gamma+2 \dot{\beta} \chi+2 \beta \dot{\chi}=0,
\end{aligned}
$$

and the EOMs, emerging from the equivalent Lagrangian $L_{\bar{B}}$ (that are different from the above EOMs) are as follows:

$$
\begin{array}{ll}
\dot{e}-2 \beta \bar{\beta}-2 \bar{b}=0, & \bar{\beta} \bar{b}-i \dot{\bar{c}} \chi+e \dot{\bar{\beta}}+\beta \bar{\beta}^{2}-\bar{c} \gamma=0, \\
\dot{\bar{b}}=\frac{p^{2}}{2}+2 \gamma \chi-2 \beta \dot{\bar{\beta}}, & e \dot{\beta}+\dot{e} \beta-\bar{b} \beta-i \dot{c} \chi+\gamma c-\beta^{2} \bar{\beta}=0 .
\end{array}
$$

We note that the expressions for $Q_{b}^{(1)}$ and $Q_{a b}^{(1)}$ have been obtained from the direct use of the Noether theorem related with the continuous symmetries. It can be checked explicitly that the following

$$
\begin{array}{ll}
s_{b} Q_{b}=-i\left\{Q_{b}, Q_{b}\right\}=0 & \Longrightarrow \quad Q_{b}^{2}=0 \\
s_{a b} Q_{a b}=-i\left\{Q_{a b}, Q_{a b}\right\}=0 & \Longrightarrow \quad Q_{a b}^{2}=0 \\
s_{b} Q_{a b}=-i\left\{Q_{a b}, Q_{b}\right\}=0 \quad \Longrightarrow \quad s_{a b} Q_{b}=-i\left\{Q_{b}, Q_{a b}\right\}=0
\end{array}
$$

are true if we choose the specific set of the above (anti-)BRST charges, namely;

$$
\begin{aligned}
& Q_{b} \equiv Q_{b}^{(2)}=\frac{1}{2} c p^{2}+b \dot{c}+\beta \bar{\beta} \dot{c}+i \beta e \gamma+\frac{1}{2} \beta(p \cdot \psi)+2 \beta^{2} \bar{\beta} \chi+2 b \beta \chi, \\
& Q_{a b} \equiv Q_{a b}^{(2)}=\frac{1}{2} \bar{c} p^{2}-\bar{b} \dot{\bar{c}}-\bar{\beta} \beta \dot{\bar{c}}+i \bar{\beta} e \gamma+\frac{1}{2} \bar{\beta}(p \cdot \psi)-2 \bar{\beta}^{2} \beta \chi-2 \bar{b} \bar{\beta} \chi
\end{aligned}
$$

from the two expressions for $Q_{(a) b}^{(1,2)}$ that have been quoted in Eq. (13). We would like to lay emphasis on the fact that, in the proof of absolute anticommutativity of the nilpotent 
(anti-)BRST charges (i.e. $s_{b} Q_{a b}=-i\left\{Q_{a b}, Q_{b}\right\}=0, s_{a b} Q_{b}=-i\left\{Q_{b}, Q_{a b}\right\}=0$ ), we have to utilize the beauty and strength of the CF-type restriction: $b+\bar{b}+2 \beta \bar{\beta}=0$. As far as the nilpotency property is concerned, we discuss more about the conserved (anti-) BRST charges and thier equivalent forms in our Appendix A. In exactly similar fashion, we discuss a few relevant theoretical computations in Appendix B about the property of absolute anticommutativity of the conserved and nilpotent charges that are related with the (anti-)BRST symmetry transformations (9) and (10). We shall capture all these features of the (anti-)BRST charges (for our free scalar and massless spinning relativistic particles) in the language of (anti-)chiral supervariable approach in the forthcoming Sec. 5.

\section{3 (Anti-)Chiral Supervariable Approach: Nilpotent (Anti-)BRST Symmetries for a Scalar Particle}

We exploit here the symmetry invariant (i.e. (anti-)BRST invariant) restrictions on the (anti-)chiral supervariables to derive the off-shell nilpotent (anti-)BRST symmetry transformations (2). In this connection, first of all, we generalize the basic and auxiliary variables of the starting Lagrangian (1) onto (1, 1)-dimensional anti-chiral supermanifold as

$$
\begin{aligned}
& x_{\mu}(\tau) \longrightarrow X_{\mu}(\tau, \bar{\theta})=x_{\mu}(\tau)+\bar{\theta} R_{\mu}(\tau), \quad p_{\mu}(\tau) \longrightarrow P_{\mu}(\tau, \bar{\theta})=p_{\mu}(\tau)+\bar{\theta} S_{\mu}(\tau), \\
& e(\tau) \longrightarrow E(\tau, \bar{\theta})=e(\tau)+\bar{\theta} f_{1}(\tau), \quad c(\tau) \longrightarrow F(\tau, \bar{\theta})=c(\tau)+i \bar{\theta} B_{1}(\tau), \\
& \bar{c}(\tau) \longrightarrow \bar{F}(\tau, \bar{\theta})=\bar{c}(\tau)+i \bar{\theta} B_{2}(\tau), \quad b(\tau) \longrightarrow \tilde{B}(\tau, \bar{\theta})=b(\tau)+\bar{\theta} f_{2}(\tau),
\end{aligned}
$$

where the $(1,1)$-dimensional supermanifold is parametrized by the superspace variables $(\tau, \bar{\theta})$ and the set $\left(R_{\mu}(\tau), S_{\mu}(\tau), f_{1}(\tau), f_{2}(\tau)\right)$ consists of fermionic secondary variables and the set $\left(B_{1}(\tau), B_{2}(\tau)\right)$ is made up of the bosonic secondary variables. All these secondary variables are function of $\tau$ and they are to be determined precisely in terms of the basic and auxiliary variables of the starting Lagrangian (1) by exploiting the theoretical strength of the (anti-)BRST (i.e. quantum gauge) invariant restrictions on the supervariables (defined on the anti-chiral supermanifold).

One of the key ingredients of the (anti-)chiral superfield/supervariable approach is the requirement that all the quantum gauge (i.e. (anti-)BRST) invariant quantities must be independent of the "soul" coordinates $(\theta, \bar{\theta})$ because these Grassmannian variables (i.e. $\theta, \bar{\theta})$ are only the mathematical artifacts which can not be physically realized $\bar{q}$. We note that the following interesting quantities are BRST invariant, namely;

$$
\begin{array}{ll}
s_{b} b=0, \quad s_{b} p_{\mu}=0, & s_{b} c=0, \quad s_{b}(e \dot{c})=0, \\
s_{b}(b e+i \bar{c} \dot{c})=0, & s_{b}\left(\dot{x}_{\mu}-e p_{\mu}\right)=0,
\end{array}
$$

where, in the last entry, we have to use the physical property of a free relativistic particle for which $\dot{p}_{\mu}=0$. The above BRST-invariant quantities must be independent of $\bar{\theta}$ when

\footnotetext{
"In the older literature [28], the spacetime coordinates have been referred to as the "body" coordinates and the Grassmannian variables have been christened as the "soul" coordinates. The former can be realized physically but the latter variables are mathematical artifacts which can not be realized in the same way.
} 
these are generalized onto the (1, 1)-dimensional anti-chiral supermanifold; namely;

$$
\begin{aligned}
& \tilde{B}(\tau, \bar{\theta})=b(\tau), \quad P_{\mu}(\tau, \bar{\theta})=p_{\mu}(\tau), \quad F(\tau, \bar{\theta})=c(\tau), \\
& E(\tau, \bar{\theta}) \dot{F}(\tau, \bar{\theta})=e(\tau) \dot{c}(\tau), \\
& B(\tau, \bar{\theta}) E(\tau, \bar{\theta})+i \bar{F}(\tau, \bar{\theta}) \dot{F}(\tau, \bar{\theta})=b(\tau) e(\tau)+i \bar{c}(\tau) \dot{c}(\tau), \\
& \dot{X}_{\mu}(\tau, \bar{\theta})-E(\tau, \bar{\theta}) P_{\mu}(\tau, \bar{\theta})=\dot{x}_{\mu}(\tau)-e(\tau) p_{\mu}(\tau) .
\end{aligned}
$$

The above BRST-invariant restrictions yield the following expressions for the secondary variables in terms of the basic and auxiliary variables:

$$
\begin{array}{llrl}
R_{\mu}(\tau)=c p_{\mu}, & S_{\mu}(\tau)=0, & f_{1}(\tau)=\dot{c} \\
B_{1}(\tau)=0, & B_{2}(\tau)=b, & f_{2}(\tau)=0 .
\end{array}
$$

When we substitute these expressions into the super expansions (cf. Eq. (18)) of the antichiral supervariables, we obtain the following super expansions for the supervariables of our theory; namely;

$$
\begin{aligned}
& P_{\mu}^{(b)}(\tau, \bar{\theta})=p_{\mu}(\tau)+\bar{\theta}(0) \equiv p_{\mu}(\tau)+\bar{\theta}\left(s_{b} p_{\mu}\right), \\
& \bar{F}^{(b)}(\tau, \bar{\theta})=\bar{c}+\bar{\theta}(i b) \equiv \bar{c}(\tau)+\bar{\theta}\left(s_{b} \bar{c}\right), \\
& F^{(b)}(\tau, \bar{\theta})=c(\tau)+\bar{\theta}(0) \equiv c(\tau)+\bar{\theta}\left(s_{b} c\right), \\
& B^{(b)}(\tau, \bar{\theta})=b(\tau)+\bar{\theta}(0) \equiv b(\tau)+\bar{\theta}\left(s_{b} b\right), \\
& E^{(b)}(\tau, \bar{\theta})=e(\tau)+\bar{\theta}(\dot{c}) \equiv e(\tau)+\bar{\theta}\left(s_{b} e\right), \\
& X_{\mu}^{(b)}(\tau, \bar{\theta})=x_{\mu}(\tau)+\bar{\theta}\left(c p_{\mu}\right) \equiv x_{\mu}(\tau)+\bar{\theta}\left(s_{b} x_{\mu}\right),
\end{aligned}
$$

where the superscript $(b)$, on the supervariables, denotes the fact that these supervariables have been obtained after the application of BRST invariant restrictions (20). It is evident that we have computed all the BRST symmetry transformations $\left(s_{b}\right)$ for all the variables of the (anti-)BRST invariant Lagrangian (1) for a free massive scalar relativistic particle. The super expansions in (22) also establish a relationship between $\partial_{\bar{\theta}} \equiv \partial / \partial \bar{\theta}$ and the BRST symmetry transformation $s_{b}$ (e.g. $\partial_{\bar{\theta}} X_{\mu}^{(b)}(\tau, \bar{\theta})=s_{b} x_{\mu}, \partial_{\bar{\theta}} \bar{F}^{(b)}(\tau, \bar{\theta})=s_{b} \bar{c}$, etc.) and, hence, the nilpotency $\left(\partial_{\bar{\theta}}^{2}=0, s_{b}^{2}=0\right)$ of these operators (in the superspace and ordinary space) are also inter-related .

To obtain the anti-BRST symmetry transformations that have been quoted in (2), we consider the generalizations of the basic and auxiliary variables of the starting Lagrangian (1) onto a $(1,1)$-dimensional chiral supermanifold (parameterized by the superspace coordinates $(\tau, \theta))$ as:

$$
\begin{aligned}
& x_{\mu}(\tau) \longrightarrow X_{\mu}(\tau, \theta)=x_{\mu}(\tau)+\theta \bar{R}_{\mu}(\tau), \\
& p_{\mu}(\tau) \longrightarrow P_{\mu}(\tau, \theta)=p_{\mu}(\tau)+\theta \bar{S}_{\mu}(\tau), \\
& e(\tau) \longrightarrow E(\tau, \theta)=e(\tau)+\theta \bar{f}_{1}(\tau), \\
& c(\tau) \longrightarrow F(\tau, \theta)=c(\tau)+i \theta \bar{B}_{1}(\tau), \\
& \bar{c}(\tau) \longrightarrow \bar{F}(\tau, \theta)=\bar{c}(\tau)+i \theta \bar{B}_{2}(\tau), \\
& b(\tau) \longrightarrow \tilde{B}(\tau, \bar{\theta})=b(\tau)+\theta \bar{f}_{2}(\tau),
\end{aligned}
$$


where the secondary variables $\left(\bar{B}_{1}(\tau), \bar{B}_{2}(\tau)\right)$ are bosonic in nature and fermi-onic secondary variables of the above super expansions are: $\bar{R}_{\mu}(\tau), \bar{S}_{\mu}(\tau), \bar{f}_{1}(\tau), \bar{f}_{2}(\tau)$. These secondary variables are to be determined in terms of the basic and auxiliary variables of the starting Lagrangian (1) by exploiting the basic tenets of (anti-)chiral supervariable approach where we demand that the quantum gauge (i.e. anti-BRST) invariant quantities should be independent of the "soul" coordinate $\theta$. Towards this goal in mind, we note that the following interesting quantities $[\mid$ of the 1D toy model of a free scalar relativistic particle, namely;

$$
\begin{aligned}
& s_{a b} \bar{c}=0, \quad s_{a b} b=0, \quad s_{a b}(e \dot{\bar{c}})=0, \quad s_{a b}(b e+i \dot{\bar{c}} c)=0, \\
& s_{a b}\left(\dot{x}_{\mu}-e p_{\mu}\right)=0, \quad s_{a b} p_{\mu}=0,
\end{aligned}
$$

are anti-BRST invariant. In particular, the last but one entry in the above equation is anti-BRST invariant because we take into account the physical input for a free scalar relativistic particle where the force acting on the particle is zero (i.e. $\dot{p}_{\mu}=0$ ) which means that momentum is conserved.

We are now in the position to impose the following restrictions on the supervariables in accordance with the basic tenet of (anti-)chiral superfield approach to BRST formalism:

$$
\begin{aligned}
& \bar{F}(\tau, \theta)=\bar{c}(\tau), \quad E(\tau, \theta) \dot{\bar{F}}(\tau, \theta)=e(\tau) \dot{\bar{c}}(\tau), P_{\mu}(\tau, \theta)=p_{\mu}(\tau) \\
& \tilde{B}(\tau, \theta) E(\tau, \theta)+i \dot{\bar{F}}(\tau, \theta) F(\tau, \theta)=b(\tau) e(\tau)+i \dot{\bar{c}}(\tau) c(\tau), \\
& \dot{X}_{\mu}(\tau, \theta)-E(\tau, \theta) P_{\mu}(\tau, \theta)=\dot{x}_{\mu}(\tau)-e(\tau) p_{\mu}(\tau) \quad \tilde{B}(\tau, \theta)=b(\tau) .
\end{aligned}
$$

The above restrictions lead to the derivation of the secondary variables in terms of the basic and auxiliary variables as:

$$
\begin{aligned}
\bar{R}_{\mu}(\tau)=\bar{c} p_{\mu}, & \bar{S}_{\mu}(\tau)=0, & \bar{f}_{1}(\tau)=0 \\
\bar{B}_{2}(\tau)=0, & \bar{f}_{2}(\tau)=\dot{\bar{c}}, & \bar{B}_{1}(\tau)=-b(\tau)
\end{aligned}
$$

Thus, it is crystal clear that the secondary variables in the expansions (23) are found accurately in terms of the dynamical and auxiliary variables of Lagrangian $L_{b}$ (cf. Eq. (1)). The substitution of the above values into the super expansions (23) leads to the following

$$
\begin{aligned}
& X_{\mu}^{(a b)}(\tau, \theta)=x_{\mu}(\tau)+\theta\left(\bar{c} p_{\mu}\right) \equiv x_{\mu}(\tau)+\theta\left(s_{a b} x_{\mu}\right), \\
& P_{\mu}^{(a b)}(\tau, \theta)=p_{\mu}(\tau)+\theta(0) \equiv p_{\mu}(\tau)+\theta\left(s_{a b} p_{\mu}\right) \\
& F^{(a b)}(\tau, \theta)=c(\tau)+\theta(-i b) \equiv c(\tau)+\theta\left(s_{a b} c\right) \\
& \bar{F}^{(a b)}(\tau, \theta)=\bar{c}+\theta(0) \equiv \bar{c}(\tau)+\theta\left(s_{a b} \bar{c}\right) \\
& E^{(a b)}(\tau, \theta)=e(\tau)+\theta(\dot{\bar{c}}) \equiv e(\tau)+\theta\left(s_{a b} e\right) \\
& \tilde{B}^{(a b)}(\tau, \theta)=b(\tau)+\theta(0) \equiv b(\tau)+\theta\left(s_{a b} b\right)
\end{aligned}
$$

where the superscript $(a b)$ on the supervariables denotes that these supervariables have been obtained after the application of anti-BRST invariant restrictions (25). Moreover, we

"We would like to emphasize that the (anti-)BRST invariant quantities, listed in (24) and (19), have been obtained by the trial and error method because there is no definite rule/principle to obtain them. More such kind of quantities might exist in the theory. 
note that the coefficients of $\theta$, in the above super expansions, are nothing but the antiBRST symmetry transformations (cf. Eq. (2)) for all the basic and auxiliary variables of Lagrangian (1). We note that: $\partial_{\theta} \longleftrightarrow s_{a b}$. In other words, the translational generator $\partial_{\theta}$ along $\theta$-direction of the chiral supermanifold is connected with the anti-BRST symmetry transformations (i.e. $\partial_{\theta} F^{(a b)}(\tau, \theta)=s_{a b} c, \partial_{\theta} E^{(a b)}(\tau, \theta)=s_{a b}$ e, etc.). Hence, the nilpotency property $\left(\partial_{\theta}^{2}=0, s_{a b}^{2}=0\right)$ of both these operators are inter-connected. These nilpotency properties also imply the nilpotency of the conserved anti-BRST charges (cf. Sec. 5) because $s_{(a) b}^{2}=0 \Leftrightarrow Q_{(a) b}^{2}=0$.

\section{4 (Anti-)BRST Symmetries for a Spinning Relativis- tic Particle: (Anti-)Chiral Supervariable Approach}

In this section, we exploit the idea of (anti-)BRST invariant restrictions on the specific combination(s) of the (anti-)chiral supervariables to derive the BRST and anti-BRST symmetry transformations for a 1D free massless spinning relativistic particle. The basic concepts behind this theoretical trick is to demand that all the (anti-)BRST invariant quantities (which are physical quantities at the quantum level) should be independent of the "soul" coordinates (i.e. Grassmannian variables) $\theta$ and $\bar{\theta}$. Towards this objective in mind, first of all, we generalize all the variables of Lagrangian $L_{B}$ (cf. Eq. (6)) on the appropriately chosen (1, 1)-dimensional anti-chiral supermanifold as follows:

$$
\begin{aligned}
& x_{\mu}(\tau) \quad \longrightarrow X_{\mu}(\tau, \bar{\theta})=x_{\mu}(\tau)+\bar{\theta} R_{\mu}(\tau), \\
& \gamma(\tau) \quad \longrightarrow \quad G(\tau, \bar{\theta})=\gamma(\tau)+\bar{\theta} b_{2}(\tau), \\
& p_{\mu}(\tau) \quad \longrightarrow P_{\mu}(\tau, \bar{\theta})=p_{\mu}(\tau)+\bar{\theta} S_{\mu}(\tau), \\
& c(\tau) \quad \longrightarrow F(\tau, \bar{\theta})=c(\tau)+i \bar{\theta} B_{1}(\tau), \\
& \psi_{\mu}(\tau) \quad \longrightarrow \Psi_{\mu}(\tau, \bar{\theta})=\psi_{\mu}(\tau)+\bar{\theta} B_{\mu}(\tau), \\
& \bar{c}(\tau) \quad \longrightarrow \bar{F}(\tau, \bar{\theta})=\bar{c}(\tau)+i \bar{\theta} B_{2}(\tau), \\
& e(\tau) \quad \longrightarrow E(\tau, \bar{\theta})=e(\tau)+\bar{\theta} f_{1}(\tau), \\
& \beta(\tau) \quad \longrightarrow \tilde{\beta}(\tau, \bar{\theta})=\beta(\tau)+i \bar{\theta} f_{3}(\tau), \\
& b(\tau) \quad \longrightarrow \quad \tilde{B}(\tau, \bar{\theta})=b(\tau)+\bar{\theta} f_{2}(\tau), \\
& \bar{\beta}(\tau) \quad \longrightarrow \tilde{\bar{\beta}}(\tau, \bar{\theta})=\bar{\beta}(\tau)+i \bar{\theta} f_{4}(\tau), \\
& \chi(\tau) \quad \longrightarrow X(\tau, \bar{\theta})=\chi(\tau)+\bar{\theta} b_{1}(\tau), \\
& \bar{b}(\tau) \quad \longrightarrow \tilde{\bar{B}}(\tau, \bar{\theta})=\bar{b}(\tau)+\bar{\theta} f_{5}(\tau),
\end{aligned}
$$

where the superspace coordinates $(\tau, \bar{\theta})$ characterize the $(1,1)$-dimensional anti-chiral supermanifold and secondary variables $\left(R_{\mu}, S_{\mu}, f_{1}, f_{2}, f_{3}, f_{4}, f_{5}\right)$ are fermionic and the set $\left(B_{\mu}, b_{1}, b_{2}, B_{1}, B_{2}\right)$ is bosonic in nature. The secondary variables are to be determined in terms of the basic and auxiliary variables of the Lagrangian $L_{B}$ by exploiting the BRST invariant restrictions for the derivation of BRST symmetry transformations (10).

The basic tenet of (anti-)chiral supervariable approach demands that the BRST invariant quantities should be independent of the Grassmannian variable $\bar{\theta}$ (of the antichiral supermanifold) when they are generalized onto the (1,1)-dimensional anti-chiral 
supermanifold. It is straightforward to note that the trivially invariant quantities such as: $s_{b} b=0, s_{b} \gamma=0, s_{b} \beta=0, s_{b} p_{\mu}=0$ imply that $B(\tau, \bar{\theta})=b(\tau), G(\tau, \bar{\theta})=\gamma(\tau), \tilde{\beta}(\tau, \bar{\theta})=$ $\beta(\tau), P_{\mu}(\tau, \bar{\theta})=p_{\mu}(\tau)$. The above restrictions lead to the following trivial expressions for the secondary variables in the above anti-chiral super expansions of the supervariables (cf. Eq. (28)):

$$
f_{2}(\tau)=0, \quad b_{2}(\tau)=0, \quad f_{3}(\tau)=0, \quad S_{\mu}(\tau)=0 .
$$

In other words, we have the following super expansions

$$
\begin{aligned}
& B^{(b)}(\tau, \bar{\theta})=b(\tau)+\bar{\theta}(0)=b(\tau)+\bar{\theta}\left(s_{b} b\right), \\
& G^{(b)}(\tau, \bar{\theta})=\gamma(\tau)+\bar{\theta}(0)=\gamma(\tau)+\bar{\theta}\left(s_{b} \gamma\right), \\
& \tilde{\beta}^{(b)}(\tau, \bar{\theta})=\beta(\tau)+\bar{\theta}(0)=\beta(\tau)+\bar{\theta}\left(s_{b} \beta\right), \\
& P_{\mu}^{(b)}(\tau, \bar{\theta})=p(\tau)+\bar{\theta}(0)=\beta(\tau)+\bar{\theta}\left(s_{b} p_{\mu}\right),
\end{aligned}
$$

where the superscript $(b)$ denotes the expansions for the supervariables after the application of BRST invariant restrictions and the coefficients of $\bar{\theta}$, in the above expansions, are nothing but the BRST symmetry transformations (i.e. $s_{b} b=0, s_{b} \gamma=0, s_{b} \beta=0, s_{b} p_{\mu}=0$ ) that have been quoted in Eq. (10). In other words, we have already derived the BRST symmetry transformations for the variables $\left(b, \gamma, \beta, p_{\mu}\right)$ of our theory of a massless spinning particle.

We now concentrate on the derivation of the BRST symmetry transformations for some non-trivial variables of our 1D toy model of a free spinning relativistic particle. Towards this goal in mind, we note that the following BRST-invariant quantitie***.

$$
\begin{array}{lcc}
s_{b}(\bar{\beta} \gamma)=0, & s_{b}(b \bar{\beta}+\gamma \bar{c})=0, & s_{b}\left(\beta^{2} \bar{\beta}+c \gamma\right)=0, \\
s_{b}(\dot{c}+2 \beta \chi)=0, & s_{b}(\bar{b}+2 \beta \bar{\beta})=0, & s_{b}(e \gamma \chi+e \bar{\beta} \dot{\beta}-i \bar{\beta} \dot{c} \chi)=0, \\
s_{b}\left(\dot{x}_{\mu}-e p_{\mu}+i \chi \psi_{\mu}\right)=0, & s_{b}\left(c p_{\mu}+\beta \psi_{\mu}\right)=0,
\end{array}
$$

are very useful for us because these can be generalized onto (1, 1)-dimensional anti-chiral supermanifold and we can invoke, for the following simple case, the basic tenet of (anti-) chiral supervariable approach to BRST formalism and demand the following

$$
\tilde{\bar{\beta}}(\tau, \bar{\theta}) G^{(b)}(\tau, \bar{\theta})=\bar{\beta}(\tau) \gamma(\tau), \quad G^{(b)}(\tau, \bar{\theta})=\gamma(\tau)
$$

which implies that $f_{4}(\tau) \propto \gamma$. We choose, for the sake of brevity: $f_{4}(\tau)=\gamma(\tau)$. This yields:

$$
\bar{\beta}^{(b)}(\tau, \bar{\theta})=\bar{\beta}(\tau)+\bar{\theta}(i \gamma) \equiv \bar{\beta}(\tau)+\bar{\theta}\left(s_{b} \bar{\beta}\right) .
$$

It is clear that the coefficient of $\bar{\theta}$ is the BRST symmetry transformation on $\bar{\beta}$ (cf. Eq. (10)). The above equation (33) would be used in the following equality due to our observation in Eq. (31) that $s_{b}(b \bar{\beta}+\gamma \bar{c})=0$, namely;

$$
\tilde{B}^{(b)}(\tau, \bar{\theta}) \bar{\beta}^{(b)}(\tau, \bar{\theta})+G^{(b)}(\tau, \bar{\theta}) \bar{F}(\tau, \bar{\theta})=b(\tau) \bar{\beta}(\tau)+\gamma(\tau) \bar{c}(\tau),
$$

** It should be noted that $s_{b}\left(\dot{x}_{\mu}-e p_{\mu}+i \chi \psi_{\mu}=0\right)$ is valid only when we take the on-shell conditions: $\dot{p}_{\mu}=0, \dot{\psi}_{\mu}=\chi p_{\mu}$ where $\dot{p}_{\mu}=0$ implies the free motion of the particle where the force acting on it is zero and $\dot{\psi}_{\mu}=\chi p_{\mu}$ implies $p \cdot \dot{\psi}=\chi p^{2} \approx 0$ which basically provides the connection between the constraints $\left(p^{2} \approx 0, p \cdot \psi \approx 0\right)$ on our system of a free massless spinning particle where we have also taken $\dot{p}_{\mu}=0$. 
which leads to the derivation of $B_{2}(\tau)=b(\tau)$ where we have used the expansion of $\bar{F}(\tau, \bar{\theta})$ from (28) and taken the inputs: $G^{(b)}(\tau, \bar{\theta})=\gamma(\tau), \tilde{B}^{(b)}(\tau, \bar{\theta})=b(\tau)$ (cf. Eq. (30)). Finally, we obtain the following super expansion for the supervariable $\bar{F}(\tau, \bar{\theta})$ :

$$
\bar{F}^{(b)}(\tau, \bar{\theta})=\bar{c}(\tau)+\bar{\theta}(i b(\tau)) \equiv \bar{c}(\tau)+\bar{\theta}\left(s_{b} \bar{c}\right) .
$$

The above expansion leads to $s_{b} \bar{c}=i b$ (cf. Eq. (10)) as the BRST symmetry transformation on the variable $\bar{c}(\tau)$. Similar sets of exercises lead to the derivation of secondary variables of the super expansions (28) in terms the basic and auxiliary variables of BRST invaraint Lagrangian $L_{B}$ (for the free motion of a massless spinning relativistic particle) as:

$$
\begin{aligned}
& B_{1}=-\beta^{2}, \quad B_{\mu}=i \beta p_{\mu}, \quad b_{1}=i \dot{\beta}, \quad f_{5}=-2 i \beta \gamma, \\
& f_{1}=\dot{c}+2 \beta \chi, \quad R_{\mu}=c p_{\mu}+\beta \psi_{\mu} \text {. }
\end{aligned}
$$

We would like to lay emphasis on the fact that we have freely used the force-free condition $\left(\dot{p}_{\mu}=0\right)$ and the on-shell conditions (e.g. $\left.\dot{\psi}_{\mu}=\chi p_{\mu}\right)$ in the derivation of the last entry in the above equation. The substitution of all these values of the secondary variables (in terms of the basic auxiliary variables of the Lagrangian $L_{B}$ ) into the super expansions of all the supervariables (28) leads to the following expansions (with BRST symmetry transformations $s_{b}$ as given in Eq. (10)):

$$
\begin{aligned}
& X_{\mu}^{(b)}(\tau, \bar{\theta})=x_{\mu}(\tau)+\bar{\theta}\left(c p_{\mu}+\beta \psi_{\mu}\right) \equiv x_{\mu}(\tau)+\bar{\theta}\left(s_{b} x_{\mu}\right), \\
& P_{\mu}^{(b)}(\tau, \bar{\theta})=p_{\mu}(\tau)+\bar{\theta}(0) \equiv p_{\mu}(\tau)+\bar{\theta}\left(s_{b} p_{\mu}\right), \\
& \Psi_{\mu}^{(b)}(\tau, \bar{\theta})=\psi_{\mu}(\tau)+\bar{\theta}\left(i \beta p_{\mu}\right) \equiv \psi_{\mu}(\tau)+\bar{\theta}\left(s_{b} \psi_{\mu}\right), \\
& F^{(b)}(\tau, \bar{\theta})=c(\tau)+\bar{\theta}\left(-i \beta^{2}\right) \equiv c(\tau)+\bar{\theta}\left(s_{b} c\right), \\
& \bar{F}^{(b)}(\tau, \bar{\theta})=\bar{c}(\tau)+\bar{\theta}(i b) \equiv \bar{c}(\tau)+\bar{\theta}\left(s_{b} \bar{c}\right), \\
& \tilde{B}^{(b)}(\tau, \bar{\theta})=b(\tau)+\bar{\theta}(0) \equiv b(\tau)+\bar{\theta}\left(s_{b} b\right), \\
& \tilde{\bar{B}}^{(b)}(\tau, \bar{\theta})=\bar{b}(\tau)+\bar{\theta}(-2 i \beta \gamma) \equiv \bar{b}(\tau)+\bar{\theta}\left(s_{b} \bar{b}\right), \\
& G^{(b)}(\tau, \bar{\theta})=\gamma(\tau)+\bar{\theta}(0) \equiv \gamma(\tau)+\bar{\theta}\left(s_{b} \gamma\right), \\
& E^{(b)}(\tau, \bar{\theta})=e(\tau)+\bar{\theta}(\dot{c}+2 \beta \chi) \equiv e(\tau)+\bar{\theta}\left(s_{b} e\right), \\
& \tilde{\beta}^{(b)}(\tau, \bar{\theta})=\beta(\tau)+\bar{\theta}(0) \equiv \beta(\tau)+\bar{\theta}\left(s_{b} \beta\right), \\
& \tilde{\bar{\beta}}(b)(\tau, \bar{\theta})=\bar{\beta}(\tau)+\bar{\theta}(i \gamma) \equiv \bar{\beta}(\tau)+\bar{\theta}\left(s_{b} \bar{\beta}\right), \\
& X^{(b)}(\tau, \bar{\theta})=\chi(\tau)+\bar{\theta}(i \dot{\beta}) \equiv \chi(\tau)+\bar{\theta}\left(s_{b} \chi\right) .
\end{aligned}
$$

We note that we have derived all the BRST symmetry transformations (cf. Eq. (10)) as the coefficients of $\bar{\theta}$ in the above total super expansions for our present $1 \mathrm{D}$ theory when it is generalized onto $(1,1)$-dimensional anti-chiral supermanifold. A close look at (37) shows that we have a relationship ${ }^{\dagger}: \partial_{\bar{\theta}} \longleftrightarrow s_{b}$. In other words, there is a deep connection between the BRST symmetry transformation $s_{b}$ in the ordinary 1D space and the geometrical quantity (i.e. translational generator $\partial_{\bar{\theta}}$ ) on the $(1,1)$-dimensional antichiral supermanifold (parameterized by $(\tau, \bar{\theta})$ ).

\footnotetext{
${ }^{\dagger}$ We have taken the partial derivative $\partial_{\bar{\theta}}$ because the $(1,1)$-dimensional (anti-)chiral supermanifold is parameterized by superspace coordinates $(\tau, \bar{\theta})$ which are two in numbers.
} 
We can concentrate now on the derivation of the nilpotent anti-BRST symmetry transformations (cf. Eq. (9)) by exploiting the beauty and strength of the (anti-)chiral supervariable formalism where we demand that all the anti-BRST invariant quantities (i.e. all the "physical" quantities at the quantum level) must be independent of the "soul" coordinate $\theta$ of the $(1,1)$-dimensional chiral supermanifold on which all the variables of the Lagrangian $L_{\bar{B}}$ (cf. Eq. (6)) are generalized as:

$$
\begin{aligned}
& x_{\mu}(\tau) \quad \longrightarrow X_{\mu}(\tau, \theta)=x_{\mu}(\tau)+\theta \bar{R}_{\mu}(\tau), \\
& \gamma(\tau) \quad \longrightarrow \quad G(\tau, \theta)=\gamma(\tau)+\theta \bar{b}_{2}(\tau), \\
& p_{\mu}(\tau) \quad \longrightarrow P_{\mu}(\tau, \theta)=p_{\mu}(\tau)+\theta \bar{S}_{\mu}(\tau), \\
& c(\tau) \quad \longrightarrow F(\tau, \theta)=c(\tau)+i \theta \bar{B}_{1}(\tau), \\
& \psi_{\mu}(\tau) \quad \longrightarrow \Psi_{\mu}(\tau, \theta)=\psi_{\mu}(\tau)+\theta \bar{B}_{\mu}(\tau), \\
& \bar{c}(\tau) \quad \longrightarrow \bar{F}(\tau, \theta)=\bar{c}(\tau)+i \theta \bar{B}_{2}(\tau), \\
& e(\tau) \quad \longrightarrow E(\tau, \theta)=e(\tau)+\theta \bar{f}_{1}(\tau), \\
& \beta(\tau) \quad \longrightarrow \tilde{\beta}(\tau, \theta)=\beta(\tau)+i \theta \bar{f}_{3}(\tau), \\
& b(\tau) \quad \longrightarrow \tilde{B}(\tau, \theta)=b(\tau)+\theta \bar{f}_{2}(\tau), \\
& \bar{\beta}(\tau) \quad \longrightarrow \bar{\beta}(\tau, \theta)=\bar{\beta}(\tau)+i \theta \bar{f}_{4}(\tau), \\
& \chi(\tau) \quad \longrightarrow X(\tau, \theta)=\chi(\tau)+\theta \bar{b}_{1}(\tau), \\
& \bar{b}(\tau) \quad \longrightarrow \tilde{\bar{B}}(\tau, \theta)=\bar{b}(\tau)+\theta \bar{f}_{5}(\tau),
\end{aligned}
$$

where the present $(1,1)$-dimensional chiral supermanifold is parameterized by the superspace variable $(\tau, \theta)$ and the secondary variables, on the r.h.s. of the above super expansions, are fermionic (i.e. $\bar{R}_{\mu}(\tau), \bar{S}_{\mu}(\tau), \bar{f}_{1}(\tau), \bar{f}_{2}(\tau), \bar{f}_{3}(\tau), \bar{f}_{4}(\tau), \bar{f}_{5}(\tau)$ ) as well as bosonic $\left(\bar{B}_{\mu}(\tau), \bar{b}_{1}(\tau), \bar{b}_{2}(\tau), \bar{B}_{1}(\tau), \bar{B}_{2}(\tau)\right)$ in nature. These secondary variables would be determined by exploiting the idea of anti-BRST invariant restrictions on the supervariables.

One of the fundamental concepts behind supervariable/superfield approach to BRST formalism is the requirement that all the (anti-)BRST invariant quantities should be independent of the Grassmannian variables when they are generalized onto appropriately chosen (anti-)chiral supermanifolds. It is straightforward to note that the trivial antiBRST invariant quantities such as: $s_{a b} \bar{b}=0, s_{a b} \gamma=0, s_{a b} \bar{\beta}=0, s_{a b} p_{\mu}=0$ imply that $\tilde{\bar{B}}(\tau, \theta)=\bar{b}(\tau), G(\tau, \theta)=\gamma(\tau), \tilde{\bar{\beta}}(\tau, \theta)=\bar{\beta}(\tau), P_{\mu}(\tau, \theta)=p_{\mu}(\tau)$. These restrictions, in turn, imply that the secondary variables of the above chiral supervariables, in the expansions (38), are zero. Thus, we have the following trivial values of the secondary variables:

$$
\bar{f}_{5}(\tau)=0, \quad \bar{b}_{2}(\tau)=0, \quad \bar{f}_{4}(\tau)=0, \quad \bar{S}_{\mu}(\tau)=0
$$

As a consequence of the above, we have the following chiral super expansions

$$
\begin{aligned}
& \tilde{\bar{B}}^{(a b)}(\tau, \theta)=\bar{b}(\tau)+\theta(0) \equiv \bar{b}(\tau)+\theta\left(s_{a b} \bar{b}\right), \\
& G^{(a b)}(\tau, \theta)=\gamma(\tau)+\theta(0) \equiv \gamma(\tau)+\theta\left(s_{a b} \gamma\right), \\
& \tilde{\bar{\beta}}^{(a b)}(\tau, \theta)=\bar{\beta}(\tau)+\theta(0) \equiv \bar{\beta}(\tau)+\theta\left(s_{a b} \bar{\beta}\right), \\
& P_{\mu}^{(a b)}(\tau, \theta)=p_{\mu}(\tau)+\theta(0) \equiv p_{\mu}(\tau)+\theta\left(s_{a b} p_{\mu}\right),
\end{aligned}
$$


where the superscript $(a b)$ denotes the chiral super expansions of the chiral supervariables after the application of the anti-BRST invariant restrictions. We observe that the coefficients of $\theta$, in the above expansions, are nothing but the anti-BRST symmetry transformations (9) for the variables $\left(\bar{b}, \gamma, \bar{\beta}, p_{\mu}\right)$ of our theory which is described by the Lagrangian $L_{\bar{B}}$ (cf. Eq. (6)). We shall exploit the expansions (40) for our further discussions.

To utilize the potential and power of the (anti-)chiral supervariable approach to BRST formalism, it is very important for us to obtain the useful (anti-)BRST invariant quantities because it is primarily these quantities that are generalized onto appropriately chosen supermanifold where we demand the (anti-)BRST invariant (i.e. quantum gauge invariant) restrictions on the supervariables which lead to the derivation of secondary variables in terms of the basic and auxiliary variables of the specifically chosen Lagrangian of the theory. In this context, we note that the following useful and interesting anti-BRST invariant quantities

$$
\begin{array}{lc}
s_{a b}(\beta \gamma)=0, \quad s_{a b}(\bar{b} \beta-\gamma c)=0, & s_{a b}\left(\beta \bar{\beta}^{2}-\bar{c} \gamma\right)=0, \\
s_{a b}(\dot{\bar{c}}+2 \bar{\beta} \chi)=0, & s_{a b}(b+2 \beta \bar{\beta})=0, \\
s_{a b}\left(\dot{x}_{\mu}-e p_{\mu}+i \chi \psi_{\mu}\right)=0, & s_{a b}\left(\bar{c} p_{\mu}+\bar{\beta} \psi_{\mu}\right)=0,
\end{array}
$$

are of paramount importance to us because these can be generalized onto the $(1,1)$ dimensional chiral supermanifold in terms of the chiral supervariables which would be subjected to the restrictions that these quantities must be independent of the Grassmannian variable $\theta$. To elaborate on it, we start off with a simple supervariable restriction where we demand that

$$
\tilde{\beta}(\tau, \theta) G^{(a b)}(\tau, \theta)=\beta(\tau) \gamma(\tau),
$$

where $\tilde{\beta}(\tau, \theta)$ and $G^{(a b)}(\tau, \theta)$ are given in expansions (38) and (40), respectively, which have to be utilized in our equation (42). We observe that the above equality leads to: $\bar{f}_{3}(\tau) \propto \gamma$. We choose for the sake of brevity: $\bar{f}_{3}(\tau)=-\gamma$ (which is different from our earlier choice $f_{4}(\tau)=+\gamma$ in the context of the derivation of BRST symmetry transformation for $\left.\bar{\beta}(\tau)\right)$. This choice immediately implies that we have derived the following:

$$
\tilde{\beta}^{(a b)}(\tau, \theta)=\beta(\tau)+\theta(-i \gamma) \equiv \beta(\tau)+\theta\left(s_{a b} \beta\right) .
$$

The above super expansion states clearly that we have already derived the anti-BRST symmetry transformation $\left(s_{a b} \beta=-i \gamma\right)$ as the coefficient of $\theta$ (cf. Eq. (9)). We utilize the above expansions (cf. Eqs. (40), (43)) in the anti-BRST invariant quantity $\left[s_{a b}(\bar{b} \beta-\gamma c)=\right.$ $0]$ where we also take the help of $\tilde{\bar{B}}^{(a b)}(\tau, \theta)=\bar{b}(\tau)+\theta(0)$ from Eq. (40). Finally, we have the following equality (which is nothing but an anti-BRST invariant restriction):

$$
\tilde{\bar{B}}^{(a b)}(\tau, \theta) \tilde{\beta}^{(a b)}(\tau, \theta)-G^{(a b)}(\tau, \theta) F(\tau, \theta)=\bar{b}(\tau) \beta(\tau)-\gamma(\tau) c(\tau) .
$$

We know that $\tilde{\bar{B}}^{(a b)}(\tau, \theta)=\bar{b}(\tau), G^{(a b)}(\tau, \theta)=\gamma(\tau)$ and the expansion for $\tilde{\beta}^{(a b)}(\tau, \theta)$ is given in Eq. (43). Using these inputs and super expansion for $F(\tau, \theta)$ from Eq. (38), it is evident that $\bar{B}_{1}(\tau)=\bar{b}$. Thus, we have obtained the following super expansion, namely;

$$
F^{(a b)}(\tau, \theta)=c(\tau)+\theta(i \bar{b}) \equiv c(\tau)+\theta\left(s_{a b} c(\tau)\right)
$$


where the superscript $(a b)$ denotes the super expansion of the supervariable $F(\tau, \theta)$ after the application of anti-BRST invariant restrictions (cf. Eq. (41) for details).

We have derived the anti-BRST symmetry transformations for the variables $\beta(\tau)$ and $c(\tau)$ in the above equations (43) and (45), respectively. This exercise can be repeated with all the other anti-BRST invariant restrictions that have been listed in equation (41). Ultimately, we obtain the following expressions for the rest of the secondary variables in terms of the basic and auxiliary variables of $L_{\bar{B}}$ (cf. Eq. (6)), namely;

$$
\begin{aligned}
& \bar{R}_{\mu}=\bar{c} p_{\mu}+\bar{\beta} \psi_{\mu}, \quad \bar{B}_{\mu}=i \bar{\beta} p_{\mu} \quad \bar{f}_{1}=\dot{\bar{c}}+2 \bar{\beta} \chi \\
& \bar{b}_{1}=i \dot{\bar{\beta}}, \quad \bar{B}_{2}=-\bar{\beta}^{2}, \quad \bar{f}_{5}=2 i \bar{\beta} \gamma .
\end{aligned}
$$

Substitution of the secondary variables into the super expansions (38) and taking the help of super expansions (40), (43) and (45), we write the final super expansions for all the supervariables of our theory as

$$
\begin{aligned}
& X_{\mu}^{(a b)}(\tau, \theta)=x_{\mu}(\tau)+\theta\left(\bar{c} p_{\mu}+\bar{\beta} \psi_{\mu}\right) \equiv x_{\mu}(\tau)+\theta\left(s_{a b} x_{\mu}\right), \\
& P_{\mu}^{(a b)}(\tau, \theta)=p_{\mu}(\tau)+\theta(0) \equiv p_{\mu}(\tau)+\theta\left(s_{a b} p_{\mu}\right), \\
& \Psi_{\mu}^{(a b)}(\tau, \theta)=\psi_{\mu}(\tau)+\theta\left(i \bar{\beta} p_{\mu}\right) \equiv \psi_{\mu}(\tau)+\theta\left(s_{a b} \psi_{\mu}\right), \\
& F^{(a b)}(\tau, \theta)=c(\tau)+\theta(i \bar{b}) \equiv c(\tau)+\theta\left(s_{a b} c\right), \\
& \bar{F}^{(a b)}(\tau, \theta)=\bar{c}(\tau)+\theta\left(-i \bar{\beta}^{2}\right) \equiv \bar{c}(\tau)+\theta\left(s_{a b} \bar{c}\right), \\
& \tilde{B}^{(a b)}(\tau, \theta)=b(\tau)+\theta(2 i \bar{\beta} \gamma) \equiv b(\tau)+\theta\left(s_{a b} b\right), \\
& \tilde{\bar{B}}^{(a b)}(\tau, \theta)=\bar{b}(\tau)+\theta(0) \equiv \bar{b}(\tau)+\theta\left(s_{a b} \bar{b}\right), \\
& G^{(a b)}(\tau, \theta)=\gamma(\tau)+\theta(0) \equiv \gamma(\tau)+\theta\left(s_{a b} \gamma\right), \\
& E^{(a b)}(\tau, \theta)=e(\tau)+\theta(\dot{\bar{c}}+2 \bar{\beta} \chi) \equiv e(\tau)+\theta\left(s_{a b} e\right), \\
& \tilde{\beta}^{(a b)}(\tau, \theta)=\beta(\tau)+\theta(-i \gamma) \equiv \beta(\tau)+\theta\left(s_{a b} \beta\right), \\
& \tilde{\bar{\beta}}^{(a b)}(\tau, \theta)=\bar{\beta}(\tau)+i \theta(0) \equiv \bar{\beta}(\tau)+\theta\left(s_{a b} \bar{\beta}\right), \\
& X^{(a b)}(\tau, \theta)=\chi(\tau)+\theta(i \overline{\bar{\beta}}) \equiv \chi(\tau)+\theta\left(s_{a b} \chi\right),
\end{aligned}
$$

where the superscript $(a b)$ on supervariables denotes the super expansions of the supervariables after the application of the anti-BRST invariant restrictions (41). It is now obvious that we have derived all the anti-BRST symmetry transformation of Eq. (9) as the coefficients of $\theta$ in the expansions (47). We note that we have also obtained a mapping $s_{a b} \longleftrightarrow \partial_{\theta}$ which states that the anti-BRST symmetry transformation $\left(s_{a b}\right)$ in the ordinary 1D space is deeply connected with the translational generator $\left(\partial_{\theta}\right)$ on the $(1,1)$-dimensional chiral supermanifold. Hence, their nilpotency properties $\left(s_{a b}^{2}=0, \partial_{\theta}^{2}=0\right)$ in the superspace (of the chiral supermanifold) and ordinary space (of the 1D flat spacetime manifold) are also inter-related.

\section{5 (Anti-)BRST Charges: Off-Shell Nilpotency and Absolute Anticommutativity Properties}

In this section, we take up the cases of both: a free massive scalar as well as a massless spinning relativistic particles and capture the nilpotency and absolute anticommutativity 
of their (anti-)BRST charges within the framework of (anti-)chiral supervariable approach to BRST formalism. First of all, we take up the case of a massive free scalar relativistic particle and focus on the nilpotent (anti-) BRST charges that have been expressed in Eq. (4). In particular, we concentrate on: $Q_{b}=b \dot{c}-\dot{b} c$ and $Q_{a b}=b \dot{\bar{c}}-\dot{b} \bar{c}$. It can be checked that these charges can be expressed in terms of the (anti-)chiral supervariables and other mathematical quantities (that are defined on the (1,1)-dimensional (anti-)chiral supermanifolds) as:

$$
\begin{aligned}
Q_{b} & =\frac{\partial}{\partial \bar{\theta}}\left[i \dot{\bar{F}}^{(b)}(\tau, \bar{\theta}) F^{(b)}(\tau, \bar{\theta})-i \bar{F}^{(b)}(\tau, \bar{\theta}) \dot{F}^{(b)}(\tau, \bar{\theta})\right] \\
& \equiv \int d \bar{\theta}\left[i \dot{\bar{F}}^{(b)}(\tau, \bar{\theta}) F^{(b)}(\tau, \bar{\theta})-i \bar{F}^{(b)}(\tau, \bar{\theta}) \dot{F}^{(b)}(\tau, \bar{\theta})\right], \\
Q_{a b} & =\frac{\partial}{\partial \theta}\left[i \bar{F}^{(a b)}(\tau, \theta) \dot{F}^{(a b)}(\tau, \theta)-i \dot{\bar{F}}^{(a b)}(\tau, \theta) F^{(a b)}(\tau, \theta)\right] \\
& \equiv \int d \theta\left[i \bar{F}^{(a b)}(\tau, \theta) \dot{F}^{(a b)}(\tau, \theta)-i \dot{\bar{F}}^{(a b)}(\tau, \theta) F^{(a b)}(\tau, \theta)\right],
\end{aligned}
$$

where we have utilized the derivatives $\left(\partial_{\theta}, \partial_{\bar{\theta}}\right)$ as well as differentials $(d \theta, d \bar{\theta})$ along with the supervariables (22) and (27) that have been derived after the application of the BRST and anti-BRST invariant restrictions (cf. Eqs. (20) and (25)) on the (anti-)chiral supervariables. It is evident that we have the following trivial equalities:

$$
\partial_{\bar{\theta}} Q_{b}=0, \quad \partial_{\theta} Q_{a b}=0 \quad \Longleftrightarrow \quad \partial_{\bar{\theta}}^{2}=\partial_{\theta}^{2}=0
$$

In other words, we note that the application of the derivatives w.r.t. Grassmannian variables $(\bar{\theta}) \theta$ (which characterize the (anti-)chiral supermanifolds) on the fermionic (i.e. offshell nilpotent) BRST and anti-BRST charges leads to zero result due to the nilpotency of the translational generators $\left(\partial_{\bar{\theta}}\right) \partial_{\bar{\theta}}$ along the $(\bar{\theta}) \theta$-directions of the (anti-)chiral supermanifolds.

The consequences of our observations in (48), (49) and (50) become very transparent when we express these in the ordinary space with the help of the mappings: $s_{b} \longleftrightarrow \partial_{\bar{\theta}}$ and $s_{a b} \longleftrightarrow \partial_{\theta}$. In other words, we observe the following interesting relationships, namely;

$$
\begin{aligned}
& Q_{b}=s_{b}[i \dot{\bar{c}} c-i \bar{c} \dot{c}] \quad \Longleftrightarrow \quad s_{b} Q_{b}=0 \quad \Longleftrightarrow \quad s_{b}^{2}=0, \\
& Q_{a b}=s_{a b}[i \bar{c} \dot{c}-i \dot{\bar{c}} c] \quad \Longleftrightarrow \quad s_{a b} Q_{a b}=0 \Longleftrightarrow s_{a b}^{2}=0 \text {, } \\
& s_{b} Q_{b}=0 \Longrightarrow-i\left\{Q_{b}, Q_{b}\right\}=0 \quad \Longrightarrow \quad Q_{b}^{2}=0 \quad \Longleftrightarrow \quad s_{b}^{2}=0 \text {, } \\
& s_{a b} Q_{a b}=0 \Longrightarrow-i\left\{Q_{a b}, Q_{a b}\right\}=0 \Longrightarrow \quad Q_{a b}^{2}=0 \quad \Longleftrightarrow \quad s_{a b}^{2}=0 \text {, }
\end{aligned}
$$

which demonstrate that the nilpotency of the (anti-)BRST symmetries (i.e. $s_{(a) b}^{2}=0$ ) implies the nilpotency of the (anti-)BRST charges (i.e. $\left.Q_{(a) b}^{2}=0\right)$. In other words, we note that the nilpotency of the translational generators $\partial_{\bar{\theta}}^{2}=0, \partial_{\theta}^{2}=0$ (within the framework of (anti-)chiral supervariable approach) is intimately connected with the nilpotency of the (anti-)BRST symmetries and corresponding charges in the ordinary space. This statement is corroborated by our observations in (48), (49), (50) and (51) where the (anti-) 
BRST symmetries and corresponding charges in the ordinary space are connected with the translational generators $\left(\partial_{\theta}, \partial_{\bar{\theta}}\right)$ on the $(1,1)$-dimensional (anti-)chiral supermanifolds.

We dwell a bit on the property of absolute anticommutativity of the off-shell nilpotent (anti-)BRST charges which is one of the key and novel observations of our present investigation. It can be checked, in the context of expansions in Eqs. (22) and (27), that the conserved (anti-)BRST charges can be also expressed as

$$
\begin{aligned}
Q_{a b} & =\frac{\partial}{\partial \bar{\theta}}\left[-i \bar{F}^{(b)}(\tau, \bar{\theta}) \dot{\bar{F}}^{(b)}(\tau, \bar{\theta})\right] \equiv \int d \bar{\theta}\left[-i \bar{F}^{(b)}(\tau, \bar{\theta}) \dot{\bar{F}}^{(b)}(\tau, \bar{\theta})\right], \\
Q_{b} & =\frac{\partial}{\partial \theta}\left[i F^{(a b)}(\tau, \theta) \dot{F}^{(a b)}(\tau, \theta)\right] \equiv \int d \theta\left[i F^{(a b)}(\tau, \theta) \dot{F}^{(a b)}(\tau, \theta)\right],
\end{aligned}
$$

where we have expressed the BRST charge $\left(Q_{b}\right)$ as the total derivative w.r.t. the translational generators $\left(\partial_{\theta}\right)$ of the chiral supermanifold and anti-BRST charge $\left(Q_{a b}\right)$ has been able to be written as the total derivative w.r.t. to the translational generator $\left(\partial_{\bar{\theta}}\right)$ of the anti-chiral supermanifold. It is now elementary to note that we have the following trivial equalities:

$$
\partial_{\theta} Q_{b}=0, \quad \partial_{\bar{\theta}} Q_{a b}=0 \quad \Longleftrightarrow \quad \partial_{\theta}^{2}=\partial_{\bar{\theta}}^{2}=0
$$

Thus, we observe that, in contrast to our results in (50), we see that the action of the translational generator $\partial_{\theta}$ of the chiral supermanifold on the BRST charge $\left(Q_{b}\right)$ and action of the derivative $\partial_{\bar{\theta}}$ (i.e. the translational generator along $\bar{\theta}$-direction of the anti-chiral supermanifold) on the anti-BRST charge turn out to produce zero result, too (primarily due to the nilpotency property: $\partial_{\theta}^{2}=\partial_{\bar{\theta}}^{2}=0$ ).

The consequences of the above observations, within the framework of (anti-)chiral supervariable approach to BRST formalism, become very transparent and clear when we express the preceding three equations in the ordinary space with the backing of our knowledge of the mappings: $s_{b} \leftrightarrow \partial_{\bar{\theta}}, s_{a b} \leftrightarrow \partial_{\theta}$. It is elementary to check that the expressions for the conserved and nilpotent (anti-)BRST charges $\left(Q_{(a) b}\right)$ can be also expressed in terms of the (anti-)BRST transformations $\left(s_{(a) b}\right)$ (in a different form than (51)) as:

$$
Q_{b}=s_{a b}(i c \dot{c}), \quad Q_{a b}=s_{b}(-i \bar{c} \dot{\bar{c}})
$$

In other words, it is quite obvious, from the above equations, that we have the following interesting relationships:

$$
\begin{aligned}
& s_{a b} Q_{b}=-i\left\{Q_{b}, Q_{a b}\right\}=0 \quad \Longleftrightarrow \quad s_{a b}^{2}=0, \\
& s_{b} Q_{a b}=-i\left\{Q_{a b}, Q_{b}\right\}=0 \quad \Longleftrightarrow \quad s_{b}^{2}=0 .
\end{aligned}
$$

Thus, we note that the absolute anticommutativity property of the nilpotent (anti-)BRST charges is hidden in the nilpotency of the (anti-)BRST symmetry transformations in the ordinary space because BRST charge can be expressed as an anti-BRST-exact quantity and anti-BRST charge can be expressed as the BRST-exact. We would like to lay emphasis on the fact that we have been able to derive equations (51) and (54) because of our knowledge of the (anti-)chiral supervariable approach to BRST formalism. 
We would like to stress on the novel observation that we have made in our present investigation. It should be clearly noted that we have considered only the (anti-)chiral super expansions of the supervariables. As a consequence, it is not obvious, at the outset, that the property of the absolute anticommutativity of the (anti-)BRST symmetries as well as their corresponding nilpotent and conserved charges would be obvious. This is due to fact that our earlier works on $\mathcal{N}=2$ supersymmetric quantum mechanical models [29-32] show that the symmetry transformations and corresponding conserved charges are not absolutely anticommuting even though we have applied the (anti-)chiral supervariable approach for the derivation of nilpotent supersymmetry transformations for a set of very interesting $\mathcal{N}=2$ supersymmetric quantum mechanical models. Thus, our observation of the absolute anticommutativity property of the conserved and nilpotent (anti-)BRST charges is a novel observation. We would like to add that we have now finally established that, in the context of BRST formalism (applied to the gauge and reparameterization invariant theories), the absolute anticommutativity of the (anti-)BRST charges ensue despite the fact that we consider only the (anti-)chiral super expansions of the supervariables/superfields within the framework of (anti-)chiral supervariable/superfield approach to BRST formalism (where the quantum gauge (i.e. (anti-)BRST) invariant restrictions on the supervariables/superfields play a decisive role). The above observation is crucial and universal.

We capture now the nilpotency of the (anti-)BRST charges $Q_{(a) b}$ that have been quoted in Eq. (17) for a massless spinning relativistic particle. By the trial and error method, we observe that these charges can be expressed in terms of the supervariables that have been obtained after the application of (anti-)BRST invariant restrictions (cf. Eqs. (37),(47)). In other words, we have the following expression for BRST charge $Q_{b}$ (cf. Eq. (17)) in terms of the geometrical quantities on the anti-chiral supermanifold, namely;

$$
\begin{aligned}
Q_{b} & =\frac{\partial}{\partial \bar{\theta}}\left[\frac{1}{2} P_{\mu}^{(b)}(\tau, \bar{\theta}) X^{\mu(b)}(\tau, \bar{\theta})+B^{(b)}(\tau, \bar{\theta}) E^{(b)}(\tau, \bar{\theta})\right. \\
& \left.+\tilde{\beta}^{(b)}(\tau, \bar{\theta}) \tilde{\bar{\beta}}^{(b)}(\tau, \bar{\theta}) E^{(b)}(\tau, \bar{\theta})\right] \\
& \equiv \int d \bar{\theta}\left[\frac{1}{2} P_{\mu}^{(b)}(\tau, \bar{\theta}) X^{\mu(b)}(\tau, \bar{\theta})+B^{(b)}(\tau, \bar{\theta}) E^{(b)}(\tau, \bar{\theta})\right. \\
& \left.+\tilde{\beta}^{(b)}(\tau, \bar{\theta}) \tilde{\bar{\beta}}^{(b)}(\tau, \bar{\theta}) E^{(b)}(\tau, \bar{\theta})\right],
\end{aligned}
$$

where all the supervariables (with superscript $(b)$ ) have been derived earlier in Sec. 4. It is clear that $\partial_{\bar{\theta}} Q_{b}=0$ due to the nilpotency $\left(\partial_{\bar{\theta}}^{2}=0\right)$ of the translational generator $\partial_{\bar{\theta}}$ along $\bar{\theta}$-direction of the $(1,1)$-dimensional anti-chiral supermanifold on which all the variables of our theory have been generalized. These observations become more transparent and lucid when we express (56) in the ordinary 1D space of the toy model of a massless spinning relativistic particle because the BRST charge can be expressed in the BRST-exact form as follows

$$
Q_{b}=s_{b}\left[\frac{1}{2} p_{\mu} x^{\mu}+b e+\beta \bar{\beta} e\right] \quad \Longleftrightarrow \quad s_{b} Q_{b}=0 \quad \Longleftrightarrow \quad s_{b}^{2}=0,
$$

which implies that the BRST charge is nilpotent of order two, namely;

$$
s_{b} Q_{b}=-i\left\{Q_{b}, Q_{b}\right\}=0 \quad \Longleftrightarrow \quad Q_{b}^{2}=0 \quad \Longleftrightarrow \quad s_{b}^{2}=0 .
$$


In other words, we observe that the nilpotency $\left(Q_{b}^{2}=0\right)$ of the BRST charge $Q_{b}$ is intimately connected with the nilpotency $\left(s_{b}^{2}=0\right)$ of the BRST symmetry transformations $s_{b}$ (cf. Eq. (10)). Within the framework of (anti-)chiral supervariable approach to BRST formalism, we note that the nilpotency of BRST charge as well as the nilpotency of the BRST symmetry transformations is deeply related with the nilpotency $\left(\partial_{\bar{\theta}}^{2}=0\right)$ of the translational generator $\partial_{\bar{\theta}}$ along $\bar{\theta}$-direction of the anti-chiral supermanifold.

We dwell a bit now on the off-shell nilpotency of the anti-BRST charge $Q_{a b}$ (cf. Eq. (17)) within the framework of the (anti-)chiral supervariable approach to BRST formalism. By the method of trial and error and keen observations, we note that the anti-BRST charge $\left(Q_{a b}\right)$ can be written on the $(1,1)$-dimensional chiral supermanifold as

$$
\begin{aligned}
Q_{a b} & =\frac{\partial}{\partial \theta}\left[\frac{1}{2} P_{\mu}^{(a b)}(\tau, \theta) X^{\mu(a b)}(\tau, \theta)-\tilde{\bar{B}}^{(a b)}(\tau, \theta) E^{(a b)}(\tau, \theta)\right. \\
& \left.-\tilde{\beta}^{(a b)}(\tau, \theta) \tilde{\bar{\beta}}^{(a b)}(\tau, \theta) E^{(a b)}(\tau, \theta)\right] \\
& \equiv \int d \theta\left[\frac{1}{2} P_{\mu}^{(a b)}(\tau, \theta) X^{\mu(a b)}(\tau, \theta)-\tilde{\bar{B}}^{(a b)}(\tau, \theta) E^{(a b)}(\tau, \theta)\right. \\
& \left.-\tilde{\beta}^{(a b)}(\tau, \theta) \tilde{\bar{\beta}}^{(a b)}(\tau, \theta) E^{(a b)}(\tau, \theta)\right]
\end{aligned}
$$

where the supervariables, with superscript $(a b)$, have been explained and derived earlier in Sec. 4. A close look at the above equation (59) immediately implies that

$$
\partial_{\theta} Q_{a b}=0 \quad \Longleftrightarrow \quad \partial_{\theta}{ }^{2}=0
$$

The above equations (59) and (60) can be translated into the ordinary space due to our knowledge $\left(s_{a b} \longleftrightarrow \partial_{\theta}\right)$ of the connection between the anti-BRST symmetry transformation $s_{a b}$ and the translational generator $\partial_{\theta}$ along $\theta$-direction of chiral $(1,1)$-dimensional supermanifold. In the ordinary 1D space, we have the following:

$$
\begin{aligned}
& Q_{a b}=s_{a b}\left[\frac{1}{2} p_{\mu} x^{\mu}-\bar{b} e-\beta \bar{\beta} e\right], \quad s_{a b} Q_{a b}=0 \quad \Longleftrightarrow \quad s_{a b}^{2}=0, \\
& s_{a b} Q_{a b}=-i\left\{Q_{a b}, Q_{a b}\right\}=0 \quad \Longleftrightarrow \quad Q_{a b}^{2}=0 \quad \Longleftrightarrow \quad s_{a b}^{2}=0 .
\end{aligned}
$$

Thus, we conclude that the nilpotency of anti-BRST charge is deeply connected with the nilpotency $\left(s_{a b}^{2}=0\right)$ of the anti-BRST symmetry transformations $s_{a b}$ which, in turn, is deeply related with the nilpotency $\left(\partial_{\theta}^{2}=0\right)$ of the translational generator $\partial_{\theta}$ along $\theta$ direction of the $(1,1)$-dimensional chiral supermanifold. In other words, the nilpotency properties of $Q_{a b}, s_{a b}$ and $\partial_{\theta}$ are intertwined together in a beautiful and meaningful manner.

Now we capture the absolute anticommutativity of the conserved BRST and anti-BRST charges $Q_{(a) b}$ (cf. Eq. (17)) which are off-shell nilpotent of order two (i.e. $\left.Q_{(a) b}^{2}=0\right)$. Towards this objective in mind, first of all, using the following equations of motion (that have been derived from $L_{B}$ ), namely;

$$
\begin{aligned}
& \frac{1}{2} p^{2}=\dot{\bar{b}}+2 \beta \dot{\bar{\beta}}-2 \gamma \chi \equiv-\dot{b}-2 \gamma \chi-2 \bar{\beta} \dot{\beta} \\
& p \cdot \psi=2 \text { ie } \gamma+2 \bar{\beta} \dot{c}-2 \beta \dot{\bar{c}}
\end{aligned}
$$


we recast the (anti-)BRST charges as:

$$
\begin{aligned}
& Q_{a b}=(b \dot{\bar{c}}-\dot{b} \bar{c})+2 i e \bar{\beta} \gamma+\bar{\beta}^{2} \dot{c}-2 \bar{c} \gamma \chi-2 \bar{c} \bar{\beta} \dot{\beta}+2 \beta \bar{\beta}^{2} \chi+2 b \bar{\beta} \chi, \\
& Q_{b}=(\dot{\bar{b}} c-\bar{b} \dot{c})+2 i e \beta \gamma-\beta^{2} \dot{\bar{c}}-2 c \gamma \chi+2 c \beta \overline{\bar{\beta}}-2 \beta^{2} \bar{\beta} \chi-2 \bar{b} \beta \chi
\end{aligned}
$$

where we have also used the CF-type restriction: $b+\bar{b}+2 \beta \bar{\beta}=0$. The above charges (which have been derived from the off-shell nilpotent charges $Q_{(a) b}$ (cf. Eq. (17))) can be written in the BRST-exact and anti-BRST-exact forms, respectively. We would like to lay emphasis on the fact that we have stated these results due to our knowledge of the (anti-)chiral supervariable approach to BRST formalism. We elaborate a bit on this aspect of our statement in the next paragraph in a clear and cogent manner.

By the method of trial and error and a keen observations of the supervariable expansions in (47), it can be checked that the BRST charge $Q_{b}$ can be written, in terms of the supervariables, as

$$
\begin{aligned}
Q_{b} & =\frac{\partial}{\partial \theta}\left[i F^{(a b)}(\tau, \theta) \dot{F}^{(a b)}(\tau, \theta)-\tilde{\beta}^{(a b)}(\tau, \theta) \tilde{\beta}^{(a b)}(\tau, \theta) E^{(a b)}(\tau, \theta)\right. \\
& \left.+2 i F^{(a b)}(\tau, \theta) \tilde{\beta}^{(a b)}(\tau, \theta) X^{(a b)}(\tau, \theta)\right] \\
& \equiv \int d \theta\left[i F^{(a b)}(\tau, \theta) \dot{F}^{(a b)}(\tau, \theta)-\tilde{\beta}^{(a b)}(\tau, \theta) \tilde{\beta}^{(a b)}(\tau, \theta) E^{(a b)}(\tau, \theta)\right. \\
& \left.+2 i F^{(a b)}(\tau, \theta) \tilde{\beta}^{(a b)}(\tau, \theta) X^{(a b)}(\tau, \theta)\right],
\end{aligned}
$$

where the supervariables with superscript $(a b)$ have been written in Eq. (47). It can be explicitly checked that the above expression (in the supervariable approach) yields the BRST charge given in Eq. (63). The absolute anticommutativity of $Q_{b}$ with $Q_{a b}$ becomes transparent when we express the above charge in the ordinary 1D space in terms of the anti-BRST symmetry transformation $s_{a b}$ (with $\partial_{\theta} \longleftrightarrow s_{a b}$ ), namely;

$$
Q_{b}=s_{a b}\left[i c \dot{c}-\beta^{2} e+2 i c \beta \chi\right] \text {. }
$$

The above equation implies the following in a straightforward manner:

$$
s_{a b} Q_{b}=-i\left\{Q_{b}, Q_{a b}\right\}=0 \quad \Longleftrightarrow \quad s_{a b}^{2}=0 .
$$

Thus, we note that the absolute anticommutativity of the BRST charge with anti-BRST charge is encoded in the nilpotency of anti-BRST symmetry transformations (i.e. $\left.s_{a b}^{2}=0\right)$. This becomes evident from the anti-BRST-exact form quoted in Eq. (65). Furthermore a close look at Eq. (64) makes it clear that $\partial_{\theta} Q_{b}=0$ due to the nilpotency $\left(\partial_{\theta}^{2}=0\right)$ of the translational generator $\partial_{\theta}$ along $\theta$-direction of the chiral supermanifold. Hence, we draw the conclusion that the absolute anticommutativity property (cf. Eq. (65)) is deeply connected with the nilpotency property $\left(s_{a b}^{2}=0, \partial_{\theta}^{2}=0\right)$ associated with antiBRST symmety transformations $s_{a b}$ and translational generator $\partial_{\theta}$.

We are now in the position to concentrate on the expression for the anti-BRST charge $Q_{a b}$ that has been quoted in (63). We can capture this expression in the terminology of (anti-)chiral supervariable approach to BRST formalism on (1, 1)-dimensional (anti-)chiral 
supermanifolds. By the trial and error method and keen observation of the expansions in (37), it can be seen that the expression for $Q_{a b}$ can be written as:

$$
\begin{aligned}
Q_{a b} & =\frac{\partial}{\partial \bar{\theta}}\left[\tilde{\beta}^{(b)}(\tau, \bar{\theta}) \tilde{\beta}^{(b)}(\tau, \bar{\theta}) E^{(b)}(\tau, \bar{\theta})-i \bar{F}^{(b)}(\tau, \bar{\theta}) \dot{\bar{F}}^{(b)}(\tau, \bar{\theta})\right. \\
& \left.-2 i \bar{F}^{(b)}(\tau, \bar{\theta}) \tilde{\bar{\beta}}^{(b)}(\tau, \bar{\theta}) X^{(b)}(\tau, \bar{\theta})\right] \\
& \equiv \int d \bar{\theta}\left[\tilde{\beta}^{(b)}(\tau, \bar{\theta}) \tilde{\beta}^{(b)}(\tau, \bar{\theta}) E^{(b)}(\tau, \bar{\theta})-i \bar{F}^{(b)}(\tau, \bar{\theta}) \dot{\bar{F}}^{(b)}(\tau, \bar{\theta})\right. \\
& \left.-2 i \bar{F}^{(b)}(\tau, \bar{\theta}) \tilde{\bar{\beta}}^{(b)}(\tau, \bar{\theta}) X^{(b)}(\tau, \bar{\theta})\right]
\end{aligned}
$$

where the supervariables with superscript $(b)$ have been derived after application of BRST invariant restrictions in (37). It is straightforward to note, from the above equation (67), that we have the following relationships:

$$
\partial_{\bar{\theta}} Q_{a b}=0 \quad \Longleftrightarrow \quad \partial_{\bar{\theta}}^{2}=0 .
$$

The absolute anticommutativity of the anti-BRST charge $\left(Q_{a b}\right)$ with the BRST charge $\left(Q_{b}\right)$ can be expressed in a very transparent manner due to our understanding of $s_{b} \longleftrightarrow \partial_{\bar{\theta}}$. Thus, in the ordinary 1D space, we have the following:

$$
Q_{a b}=s_{b}\left[\bar{\beta}^{2} e-i \bar{c} \dot{\bar{c}}-2 i \bar{c} \bar{\beta} \chi\right] \text {. }
$$

From the above equation, it is straightforward to note that we have

$$
s_{b} Q_{a b}=-i\left\{Q_{a b}, Q_{b}\right\}=s_{b}^{2}\left[\bar{\beta}^{2} e-i \bar{c} \dot{\bar{c}}-2 i \bar{c} \bar{\beta} \chi\right]=0,
$$

which demonstrates that the absolute anticommutativity of the anti-BRST charge with BRST charge is ultimately connected with the nilpotency $\left(s_{b}^{2}=0\right)$ of the BRST transformations $s_{b}$ which, in turn, is connected with the nilpotency $\left(\partial_{\bar{\theta}}^{2}=0\right)$ of the translational generator $\partial_{\bar{\theta}}$ along the Grassmannian $\bar{\theta}$-direction of the anti-chiral supermanifold (parameterized by $\tau$ and $\bar{\theta})$.

\section{Summary and Conclusions}

n our present endeavor, we have discussed two toy models (i.e. a free massive scalar relativistic particle and a free spinning relativistic particle which are the examples of a set of reparameterization invariant theories) within the framework of (anti-)chiral supervariable approach to BRST formalism and established that the absolute anticommutativity of the conserved and off-shell nilpotent (anti-)BRST charges is satisfied despite the fact that we have taken into consideration only the (anti-)chiral super expansions of the supervariables on a set of suitably chosen $(1,1)$-dimensional (anti-)chiral supermanifolds. Our results, once again, have demonstrated that the simple (but intuitive and beautiful) (anti-)chiral supervariable/superfield approach (i) to the gauge as well as the reparameterization invariant theories is good enough to capture two of the central mathematical properties (i.e. 
nilpotency and absolute anticommutativity) associated with the (anti-)BRST symmerties and corresponding conserved and charges, and (ii) to provide the geometrical meanings (see, e.g. [17-22]) to the symmetry transformations and corresponding conserved charges.

In the (anti-)chiral supervariable approach to BRST formalism, the key role is played by the quantum gauge [i.e. (anti-)BRST] invariant restrictions on the (anti-)chiral supervariables that lead to the derivation of (anti-)BRST symmetries. Furthermore, we observe that the conserved and nilpotent charges of the theory could be expressed in terms of the supervariables (that are obtained after the application of the (anti-)BRST invariant restrictions) and some other geometrical quantities (e.g. Grassmannian derivative and differentials) that are defined on the suitably chosen $(1,1)$-dimensional (anti-)chiral supermanifolds on which the reparameterization invariant theories are generalized. Ultimately, this exercise leads to the BRST and anti-BRST exact forms of the expressions for the conserved (anti-)BRST charges and this, in turn, produces the nilpotency and absolute anticommutativity of the BRST and anti-BRST charges within the framework of (anti-)chiral supervariable approach to BRST formalism. The observation of the absolute anticommutativity property of the (anti-)BRST charges is a completely novel result in our present endeavor (and earlier works [17-22]) in view of the fact that only the (anti-)chiral super expansions of the supervariables have been taken into account.

In our present endeavor, we have applied our (anti-)chiral supervariable approach to simple toy models of an ordinary scalar relativistic particle and a spinning relativistic particle and have shown the validity of absolute anticommutativity of the (anti-)BRST charges where the CF-type restriction plays a key role (in the context of supersymmetric spinning relativistic particle). The crucial point to be noted is the (non-)existence of CFtype restriction in both the toy models that have been taken into account in our present investigation. As a consequence of the above observations, we see that the Lagrangian (1) for a scalar relativistic particle respects both BRST and anti-BRST symmetries. However, there is an existence of a coupled (but equivalent) Lagrangians (cf. Eq. (6)) for the description of a free spinning relativistic particle. We note that $L_{B}$ and $L_{\bar{B}}$ (cf. Eq. (6)) have perfect BRST and anti-BRST symmetries (cf. Eqs. (11),(12)), respectively, but when one applies the anti-BRST transformations on $L_{B}$ and BRST symmetry transformations on $L_{\bar{B}}$, these Lagrangians transform to the total derivatives plus terms that vanish due to the CF-restriction [23].

As far as the off-shell nilpotency and absolute anticommutativity of the conserved BRST and anti-BRST charges are concerned, we observe that the off-shell nilpotency of BRST charge is connected with the nilpotency $\left(\partial_{\bar{\theta}}^{2}=0\right)$ of the translational generator $\left(\partial_{\bar{\theta}}\right)$ along $\bar{\theta}-$ direction of $(1,1)$-dimensional anti-chiral supermanifold as well as the nilpotency $\left(s_{b}^{2}=0\right)$ of the BRST symmetry transformations $\left(s_{b}\right)$. In exactly similar fashion, the off-shell nilpotency of anti-BRST charge is connected with the nilpotency properties $\left(\partial_{\theta}^{2}=0, s_{a b}^{2}=0\right)$ associated with the translational generator $\left(\partial_{\theta}\right)$ along $\theta$-direction of the chiral supermanifold and anti-BRST symmetry transformations $\left(s_{a b}\right)$. On the contrary, we observe that the absolute anticommutativity of the BRST charge with anti-BRST charge is connected with the nilpotency properties $\left(\partial_{\theta}^{2}=0, s_{a b}^{2}=0\right)$ of $\partial_{\theta}$ and $s_{a b}$ (cf. Eqs. (64),(65)). Further, we note that the absolute anticommutativity of the anti-BRST charge with BRST charge is connected with the nilpotency properties $\left(\partial_{\bar{\theta}}^{2}=0, s_{b}^{2}=0\right)$ of $\partial_{\bar{\theta}}$ and $s_{b}$ (cf. Eqs. (68),(69)). These latter observations, connected with the property of absolute anticommutativity, are 
completely novel results. In fact, this is one of the highlights of our present investigation where we have proven the absolute anticommutativity property of the conserved charges $d e$ spite the fact that we have taken into consideration only the (anti-)chiral super expansions for the supervariables.

It would be a challenging problem for us to apply our method of derivation of the nilpotent (anti-)BRST symmetries to some physical problems of interest in the physical four $(3+1)$-dimensions of spacetime. It would be also interesting to prove the absolute anticommutativity, nilpotency, etc., of the 4D field theoretic and/or supersymmetric models of physical interest that respect the reparameterization invariance. Such theories are, of course, the gravitational as well as (super)string/supergravity theories where the reparameterization invariance plays a decisive role. We plan to pursue all the above cited problems in the forthcoming years through our future publications [33].

Acknowledgements: Financial supports from the BHU-fellowship and DST-INSPIRE fellowship are gratefully acknowledged by S. Kumar and B. Chauhan, respectively. The present work has been carried out under the above fellowships.

\section{Appendix A: On the Nilpotent (Anti-)BRST Charges}

We comment here on the derivation of equivalent expressions for the conserved charges (cf. Eq. (17)) and their nilpotency properties by using the idea of continuous symmetry transformations and their generators. First of all, we note that the expressions for the (anti-)BRST charges (that are derived directly by using the Noether theorem) are

$$
\begin{aligned}
& Q_{a b}^{(1)}=\frac{1}{2} \bar{c} p^{2}-\bar{b} \dot{\bar{c}}+\bar{\beta}(p \cdot \psi)-\bar{\beta}^{2} \dot{c}-2 \beta \bar{\beta}^{2} \chi-2 \bar{b} \bar{\beta} \chi \\
& Q_{b}^{(1)}=\frac{1}{2} c p^{2}+b \dot{c}+\beta(p \cdot \psi)+\beta^{2} \dot{\bar{c}}+2 \beta^{2} \bar{\beta} \chi+2 b \beta \chi,
\end{aligned}
$$

where the continuous symmetry transformations (9) and (10) have been used in the following explicit expressions for the Noether conserved (anti-)BRST charges in the context of a 1D supersymmetric toy model of a spinning relativistic particle (cf. Eqs. (11),(12)):

$$
\begin{aligned}
& Q_{a b}^{(1)}=s_{a b} \phi_{i}\left(\frac{\partial L_{\bar{B}}}{\partial \phi_{i}}\right)-\frac{1}{2} \bar{c} p^{2}-\frac{1}{2} \bar{\beta}(p \cdot \psi)+\bar{b}(\dot{\bar{c}}+2 \bar{\beta} \chi), \\
& Q_{b}^{(1)}=s_{b} \phi_{i}\left(\frac{\partial L_{B}}{\partial \phi_{i}}\right)-\frac{1}{2} c p^{2}-\frac{1}{2} \beta(p \cdot \psi)-b(\dot{c}+2 \beta \chi) .
\end{aligned}
$$

In the above, we have $\phi_{i}\left(\equiv x_{\mu}, e, \bar{c}, c, b, \bar{b}, \psi_{\mu}, p_{\mu}, \beta, \bar{\beta}, \gamma\right)$ as the generic variable of the theory. If we apply directly $s_{(a) b}$ on the above Noether charges (cf. (A.1)), we obtain:

$$
s_{a b} Q_{a b}^{(1)}=\frac{1}{2} i \bar{\beta}^{2} p^{2}-i \bar{\beta}^{2} \dot{\bar{b}}+2 i \bar{\beta}^{2} \gamma \chi-2 i \bar{\beta}^{2} \beta \dot{\bar{\beta}}
$$




$$
s_{b} Q_{b}^{(1)}=\frac{1}{2} i \beta^{2} p^{2}+i \beta^{2} \dot{b}+2 i \beta^{2} \gamma \chi+2 i \beta^{2} \bar{\beta} \dot{\beta} .
$$

The above equations (with the inputs $\left.s_{b} Q_{b}^{(1)}=-i\left\{Q_{b}^{(1)}, Q_{b}^{(1)}\right\}, s_{a b} Q_{a b}^{(1)}=-i\left\{Q_{a b}^{(1)}, Q_{a b}^{(1)}\right\}\right)$ demonstrate that the conserved charges $Q_{(a) b}^{(1)}$ (cf. (A.1)) are not nilpotent of order two unless we apply the following equations of motion (derived from $L_{B}$ and $L_{\bar{B}}$ ):

$$
\dot{b}=-\frac{1}{2} p^{2}-2 \gamma \chi-2 \bar{\beta} \dot{\beta}, \quad \dot{\bar{b}}=\frac{1}{2} p^{2}+2 \gamma \chi-2 \beta \dot{\bar{\beta}}
$$

Thus, we note that the (anti-)BRST charges, derived directly from the Noether theorem, are not off-shell nilpotent despite the fact that the (anti-)BRST symmetries (quoted in Eqs. (9), (10)) are themselves off-shell nilpotent. This is the reason that the (anti-)BRST charges (A.1) have been recast in a different form using the EOMs. These appropriate offshell nilpotent charges have been quoted in Eq. (17) and have been denoted by $Q_{b}^{(2)} \equiv Q_{b}$ and $Q_{a b}^{(2)} \equiv Q_{a b}$.

We end this Appendix with the remark that there are other equivalent expressions for the conserved charges $Q_{(a) b}$ that are on-shell nilpotent. These are listed below:

$$
\begin{gathered}
Q_{a b}^{(3)} \equiv \dot{\bar{b}} \bar{c}-\bar{b} \dot{\bar{c}}-2 \bar{c} \gamma \chi+2 \bar{c} \beta \dot{\bar{\beta}}+\bar{\beta}(p \cdot \psi)-\bar{\beta}^{2} \dot{c}-2 \beta \bar{\beta}^{2} \chi-2 \bar{b} \bar{\beta} \chi, \\
Q_{a b}^{(4)} \equiv \dot{\bar{b}} \bar{c}-\bar{b} \dot{\bar{c}}+\frac{1}{2} \bar{\beta}(p \cdot \psi)-2 \bar{c} \gamma \chi-2 \bar{c} \beta \dot{\bar{\beta}}-\bar{\beta} \beta \dot{\bar{c}}-i \bar{\beta} e \gamma-2 \bar{\beta}^{2} \beta \chi-2 \bar{b} \bar{\beta} \chi, \\
Q_{b}^{(3)} \equiv b \dot{c}-\dot{b} c-2 c \gamma \chi-2 c \bar{\beta} \dot{\beta}+\beta(p \cdot \psi)+\beta^{2} \dot{\bar{c}}+2 \beta^{2} \bar{\beta} \chi+2 b \beta \chi, \\
Q_{b}^{(4)} \equiv b \dot{c}-\dot{b} c+\frac{1}{2} \beta(p \cdot \psi)-2 c \gamma \chi-2 c \bar{\beta} \dot{\beta}+\beta \bar{\beta} \dot{c}+i \beta e \gamma+2 \beta^{2} \bar{\beta} \chi+2 b \beta \chi .
\end{gathered}
$$

We can check that the above charges are nilpotent (i.e. $s_{b} Q_{b}^{(3,4)}=0$ and $s_{a b} Q_{a b}^{(3,4)}=0$ ) only when we use the EOM (A.4). We note that the above charges $Q_{b}^{(3,4)}$ and $Q_{a b}^{(3,4)}$ have been derived from (A.1) by using the appropriate EOMs that are listed in Eqs. (14) and (15). Thus, we draw the conclusion that the conserved (anti-)BRST charges $Q_{(a) b}^{(1,3,4)}$ are nilpotent only when we use the EOMs. However, the conserved (anti-)BRST charges $Q_{(a) b}^{(2)}$ are nilpotent without any use of EOMs. This is why, we have chosen these charges (in Eq. (17)) whose absolute anticommutativity property has been derived and discussed in Sec. 5.

\section{Appendix B: On the Absolute Anticommutativity Property}

In this Appendix, we dwell a bit on the property of absolute anticommuatativity of the (anti-)BRST charges that have been discussed in Sec. 2 (and Appendix A) in the context of 1D toy model of a massless spinning relativistic particle. When we apply the anti-BRST symmetry transformations on BRST charge $\left(Q_{b}\right)$ and BRST symmetry transformation on anti-BRST charge $\left(Q_{a b}\right)$ (cf. Eq. (17)), we obtain the following:

$$
s_{a b} Q_{b}=\frac{1}{2} i p^{2}(\bar{b}+\beta \bar{\beta})+i(b+\beta \bar{\beta}) \dot{\bar{b}}-2 i(b+\beta \bar{\beta}) \gamma \chi
$$




$$
\begin{gathered}
+2 i \beta(b+\beta \bar{\beta}) \dot{\bar{\beta}}-i \bar{\beta} \gamma \dot{c}-i \bar{\beta} \gamma \dot{\bar{c}}-\frac{i}{2} \gamma(p \cdot \psi), \\
s_{b} Q_{a b}=\frac{1}{2} i p^{2}(b+\beta \bar{\beta})-i(\bar{b}+\beta \bar{\beta}) \dot{b}-2 i(\bar{b}+\beta \bar{\beta}) \gamma \chi \\
2 i \bar{\beta}(\bar{b}+\beta \bar{\beta}) \dot{\beta}+i \beta \gamma \dot{\bar{c}}+i \bar{\beta} \dot{c} \gamma+\frac{i}{2} \gamma(p \cdot \psi) .
\end{gathered}
$$

Using the CF-type restriction $(b+\bar{b}+2 \beta \bar{\beta}=0)$ which implies that $\beta \bar{\beta}+b=-(\bar{b}+\beta \bar{\beta})$, we obtain the following:

$$
\begin{gathered}
s_{a b} Q_{b}=i(b+\beta \bar{\beta})\left[\dot{\bar{b}}-\frac{p^{2}}{2}+2 \beta \dot{\bar{\beta}}-2 \gamma \chi\right]-i \gamma\left[\frac{1}{2}(p \cdot \psi)-\bar{\beta} \dot{c}+\beta \dot{\bar{c}}\right], \\
s_{b} Q_{a b}=-i(\bar{b}+\beta \bar{\beta})\left[\dot{b}+\frac{p^{2}}{2}+2 \beta \dot{\bar{\beta}}+2 \gamma \chi\right]+i \gamma\left[\frac{1}{2}(p \cdot \psi)-\bar{\beta} \dot{c}+\beta \dot{\bar{c}}\right] .
\end{gathered}
$$

It is crystal clear that if we use the $\operatorname{EOMs}(\mathrm{A} .4)$ and $\frac{1}{2}(p \cdot \psi)+\beta \dot{\bar{c}}-\bar{\beta} \dot{c}=i$ e $\gamma$, we obtain the absolute anticommutativity $\left(\left\{Q_{b}, Q_{a b}\right\}=0\right)$ of the charges due to the basic relationship between the continuous symmetry transformations and the conserved and nilpotent charges as the generators in: $s_{b} Q_{a b}=-i\left\{Q_{a b}, Q_{b}\right\}=0$ and $s_{a b} Q_{b}=-i\left\{Q_{b}, Q_{a b}\right\}=0$.

To prove the absolute anticommutativity of the (anti-)BRST charges $\left(Q_{(a) b}^{(1)}\right)$ that have been derived directly by using the Noether theorem, we note that:

$$
\begin{aligned}
& s_{a b} Q_{b}^{(1)}=\frac{1}{2} i p^{2}[\bar{b}+2 \beta \bar{\beta}]+i b[\dot{\bar{b}}+2 \beta \dot{\bar{\beta}}-2 \gamma \chi] i \gamma[(p \cdot \psi)-2 \bar{\beta} \dot{c}+2 \beta \dot{\bar{c}}], \\
& s_{b} Q_{a b}^{(1)}=\frac{1}{2} i p^{2}[b+2 \beta \bar{\beta}]-i \bar{b}[\dot{b}+2 \dot{\beta} \bar{\beta}+2 \gamma \chi]+i \gamma[(p \cdot \psi)+2 \beta \dot{\bar{c}}-2 \bar{\beta} \dot{c}] .
\end{aligned}
$$

Using the equations of motion (A.4), CF-type restriction $(b+\bar{b}+2 \beta \bar{\beta}=0)$ and $(p \cdot \psi)-$ $2 \bar{\beta} \dot{c}+2 \beta \dot{\bar{c}}=2 i e \gamma$, it can be seen that

$$
\begin{gathered}
s_{a b} Q_{b}^{(1)}=-\frac{1}{2} i b p^{2}+\frac{1}{2} i b p^{2}=0 \quad \Longleftrightarrow \quad-i\left\{Q_{b}^{(1)}, Q_{a b}^{(1)}\right\}=0, \\
s_{b} Q_{a b}^{(1)}=-\frac{1}{2} i \bar{b} p^{2}+\frac{1}{2} i \bar{b} p^{2}=0 \Longleftrightarrow-i\left\{Q_{a b}^{(1)}, Q_{b}^{(1)}\right\}=0,
\end{gathered}
$$

where we have taken into account the key relationship between the symmetry generators and continuous symmetry transformations so that $s_{b} Q_{a b}^{(1)}=-i\left\{Q_{a b}^{(1)}, Q_{b}^{(1)}\right\}=0$ and $s_{a b} Q_{b}^{(1)}=-i\left\{Q_{b}^{(1)}, Q_{a b}^{(1)}\right\}=0$. In other words, we have proven the absolute anticommutativity of the (anti-)BRST charges $\left(Q_{(a) b}^{(1)}\right)$ that are derived directly by using the Noether theorem. However, we note that we have used the EOMs (15) and (14) in addition to the CF-type restriction for this proof. We draw the conclusion that we have to find out the appropriate form of the (anti-)BRST charges which could be written precisely in the BRSTexact and anti-BRST-exact forms so that the absolute anticommutativity could be proven only by using the CF-type restriction. This has been precisely and elegantly achieved (cf. Eqs. (65), (69)) in the main body of our text (cf. Sec. 5). 


\section{Appendix C: On the (Anti-)BRST Invariance}

In this Appendix, we concisely discuss the (anti-)BRST invariance of the Lagrangians $L_{\bar{B}}$ and $L_{B}$ within the framework of (anti-)chiral supervariable approach to BRST formalism. In this context, first of all, we note that the starting Lagrangian $\left(L_{0}\right)$ for our 1D toy model of spinning relativistic particle (cf. Eq. (7)) remains invariant under the (anti-)BRST symmetry transformations (cf. Eqs. (9) and (10)). It can be explicitly checked that:

$$
\begin{gathered}
s_{b} L_{0}=\frac{d}{d \tau}\left[\frac{1}{2} c p^{2}+\frac{1}{2} \beta(p \cdot \psi)\right], \\
s_{a b} L_{0}=\frac{d}{d \tau}\left[\frac{1}{2} \bar{c} p^{2}+\frac{1}{2} \bar{\beta}(p \cdot \psi)\right] .
\end{gathered}
$$

The above invariance can be captured within the framework of (anti-)chiral supervariable approach to BRST formalism. Towards this goal in mind, we generalize the Lagrangian $L_{0}$ to its counterpart super Lagrangians as

$$
\begin{gathered}
L_{0} \longrightarrow \tilde{L}_{0}^{(a b)}=p_{\mu}(\tau) \dot{X}^{\mu(a b)}(\tau, \theta)-\frac{1}{2} E^{(a b)}(\tau, \theta) p^{2}(\tau)+\frac{i}{2} \Psi_{\mu}^{(a b)}(\tau, \theta) \dot{\Psi}^{\mu(a b)}(\tau, \theta) \\
+i X^{(a b)}(\tau, \theta) p_{\mu}(\tau) \Psi^{\mu(a b)}(\tau, \theta) \\
L_{0} \longrightarrow \tilde{L}_{0}^{(b)}=p_{\mu}(\tau) \dot{X}^{\mu(b)}(\tau, \bar{\theta})-\frac{1}{2} E^{(b)}(\tau, \bar{\theta}) p^{2}(\tau) \\
+\frac{i}{2} \Psi_{\mu}^{(b)}(\tau, \bar{\theta}) \dot{\Psi}^{\mu(b)}(\tau, \bar{\theta})+i X^{(b)}(\tau, \bar{\theta}) p_{\mu}(\tau) \Psi^{\mu(b)}(\tau, \bar{\theta}),
\end{gathered}
$$

where we have taken into account $P_{\mu}^{(b)}(\tau, \bar{\theta})=p_{\mu}(\tau)$ and $P_{\mu}^{(a b)}(\tau, \theta)=p_{\mu}(\tau)$ because $p_{\mu}(\tau)$ is an (anti-)BRST invariant quantity (i.e. $\left.s_{(a) b} p_{\mu}(\tau)=0\right)$. Now it is an elementary exercise to observe that we have the following:

$$
\begin{aligned}
\frac{\partial}{\partial \theta} \tilde{L}_{0}^{(a b)} & =\frac{d}{d \tau}\left[\frac{1}{2} \bar{c} p^{2}+\frac{1}{2} \bar{\beta}(p \cdot \psi)\right], \\
\frac{\partial}{\partial \bar{\theta}} \tilde{L}_{0}^{(b)} & =\frac{d}{d \tau}\left[\frac{1}{2} c p^{2}+\frac{1}{2} \beta(p \cdot \psi)\right] .
\end{aligned}
$$

Taking into account the mapping: $s_{b} \longleftrightarrow \partial_{\bar{\theta}}, s_{a b} \longleftrightarrow \partial_{\theta}$, we can translate the above equation (C.4) into the ordinary 1D space of the toy model of a spinning relativistic particle which, ultimately, boils down to the results that have been quoted in (C.1). We would like to point out that the superscripts $(a b)$ and (b) on the super Lagrangians (C.2) and (C.3) denote the fact that these Lagrangians have been expressed in terms of the supervariables that have been obtained after the anti-BRST and BRST invariant restrictions.

We would like to capture now the (anti-)BRST invariance of the gauge-fixing and Faddeev-Popove (FP) ghost terms (from the Lagrangian $L_{\bar{B}}$ and $L_{B}$ ) within the framework of (anti-)chiral supervariable approach to BRST formalism. Towards this objective in mind, we have to focus first on $L_{B}$ where we take into account $\tilde{\beta}^{(b)}(\tau, \bar{\theta})=\beta(\tau), G^{(b)}(\tau, \bar{\theta})=$ 
$\gamma(\tau), \tilde{B}^{(b)}(\tau, \bar{\theta})=b(\tau)$. With these as inputs, we can generalize the gauge-fixing and FPghost terms (of $L_{B}$ ) in terms of appropriate supervariables as

$$
\begin{aligned}
\tilde{L}_{B}^{(g f)}+\tilde{L}_{B}^{(f p)}= & b(\tau) \dot{E}^{(b)}(\tau, \bar{\theta})+b(\tau)\left[b(\tau)+2 \beta(\tau) \tilde{\beta^{(b)}}(\tau, \bar{\theta})\right] \\
& -i \dot{\bar{F}}^{(b)}(\tau, \bar{\theta})\left[\dot{F}^{(b)}(\tau, \bar{\theta})+2 \beta(\tau) X^{(b)}(\tau, \bar{\theta})\right] \\
& +2 i \tilde{\bar{\beta}}^{(b)}(\tau, \bar{\theta}) \dot{F}^{(b)}(\tau, \bar{\theta}) X^{(b)}(\tau, \bar{\theta})+2 \beta(\tau) \gamma(\tau) \bar{F}^{(b)}(\tau, \bar{\theta}) \\
& -2 E^{(b)}(\tau, \bar{\theta})\left[\gamma(\tau) X^{(b)}(\tau, \bar{\theta})+\tilde{\bar{\beta}}^{(b)}(\tau, \bar{\theta}) \dot{\beta}(\tau)\right] \\
& +\beta^{2}(\tau) \tilde{\bar{\beta}}^{2(b)}(\tau, \bar{\theta})+2 \tilde{\bar{\beta}}^{(b)}(\tau, \bar{\theta}) F^{(b)}(\tau, \bar{\theta}) \gamma(\tau),
\end{aligned}
$$

where the superscript $(b)$ denotes the fact that we have taken into account the super expansions (37) for the supervariables of our theory. It can be checked explicitly that we have the following:

$$
\frac{\partial}{\partial \bar{\theta}}\left[\tilde{L}_{B}^{(g f)}+\tilde{L}_{B}^{(f p)}\right]=\frac{d}{d \tau}[b(\dot{c}+2 \beta \chi)] .
$$

In exactly similar fashion, we can capture the result of the application of off-shell nilpotent anti-BRST symmetry transformations on the gauge-fixing and Faddeev-Popov ghost terms of the Lagrangian $L_{\bar{B}}$. Towards this goal in mind, first of all, we take into account $G^{(a b)}(\tau, \theta)=\gamma(\tau), \tilde{\bar{B}}^{(a b)}(\tau, \theta)=\bar{b}(\tau), \tilde{\bar{\beta}}^{(a b)}(\tau, \theta)=\bar{\beta}(\tau)$. With these as inputs, we have the following form of the super Lagrangian for the gauge-fixing and FP ghost terms

$$
\begin{aligned}
\tilde{L}_{\bar{B}}^{(g f)}+ & \tilde{L}_{\bar{B}}^{(f p)}=-\bar{b}(\tau) \dot{E}^{(a b)}(\tau, \theta)+\bar{b}(\tau)\left[\bar{b}(\tau)+2 \bar{\beta}(\tau) \tilde{\beta}^{(a b)}(\tau, \theta)\right] \\
& -i \dot{\bar{F}}^{(a b)}(\tau, \theta)\left[\dot{F}^{(a b)}(\tau, \theta)+2 \tilde{\beta}^{(a b)}(\tau, \theta) X^{(a b)}(\tau, \theta)\right] \\
+2 i & \bar{\beta}(\tau) \dot{F}^{(a b)}(\tau, \theta) X^{(a b)}(\tau, \theta)+2 \tilde{\beta}^{(a b)}(\tau, \theta) \gamma(\tau) \bar{F}^{(a b)}(\tau, \theta) \\
& -2 E^{(a b)}(\tau, \theta)\left[\gamma(\tau) X^{(a b)}(\tau, \theta)-\tilde{\beta}^{(a b)}(\tau, \theta) \dot{\bar{\beta}}(\tau)\right] \\
& +\bar{\beta}^{2}(\tau) \tilde{\beta}^{2(a b)}(\tau, \theta)+2 \tilde{\beta}^{(a b)}(\tau, \theta) F^{(a b)}(\tau, \theta) \gamma(\tau),
\end{aligned}
$$

where all the supervariables with superscript $(a b)$ have been derived earlier in terms of the anti-BRST symmetry transformations $s_{a b}$ (cf. Eq. (47)). Finally, we note that封

$$
\frac{\partial}{\partial \theta}\left[\tilde{L}_{\bar{B}}^{(g f)}+\tilde{L}_{\bar{B}}^{(f p)}\right]=\frac{d}{d \tau}[-\bar{b}(\dot{\bar{c}}+2 \bar{\beta} \chi)] .
$$

We end our discussion on the (anti-)chiral supervariable approach to BRST formalism in the context of the 1D toy model of a spinning relativistic particle by the observation that sum of our results in (C.8) and (C.4) as well as (C.6) and (C.4) produce the following

$$
\frac{\partial}{\partial \theta}\left[\tilde{L}_{0}+\tilde{L}_{\bar{B}}^{(g f)}+\tilde{L}_{\bar{B}}^{(f p)}\right]=\frac{d}{d \tau}\left[\frac{1}{2} \bar{c} p^{2}+\frac{1}{2} \bar{\beta}(p \cdot \psi)-\bar{b}(\dot{\bar{c}}+2 \bar{\beta} \chi)\right],
$$

\footnotetext{
${ }_{\ddagger}$ A close look at the equations (C.6) and (C.8) demonstrates that the gauge-fixing and Faddeev-Popov ghost terms of both the Lagrangians $L_{B}$ and $L_{\bar{B}}$ are BRST and anti-BRST invariant, respectively.
} 


$$
\frac{\partial}{\partial \bar{\theta}}\left[\tilde{L}_{0}+\tilde{L}_{B}^{(g f)}+\tilde{L}_{B}^{(f p)}\right]=\frac{d}{d \tau}\left[\frac{1}{2} c p^{2}+\frac{1}{2} \beta(p \cdot \psi)+b(\dot{c}+2 \beta \chi)\right],
$$

which are nothing but the results that have been quoted in Eqs. (12) and (11) for the 1D toy model of a spinning relativistic particle in the ordinary space. Thus, we have captured the (anti-)BRST invariance of the action integrals corresponding to the Lagrangians $L_{\bar{B}}$ and $L_{B}$ for the 1D toy model of a free spinning (i.e supersymmetric) relativistic particle.

We end this Appendix with a concise discussion on the (anti-)BRST invariance (cf. Eq. (3)) of the Lagrangian $L_{b}$ (cf. Eq. (1)) for a free scalar relativistic particle. Taking the help of our super expansions in (22) and (27), it can be seen that the Lagrangian $L_{b}$ can be generalized to its counterparts super Lagrangians on the (1,1)-dimensional (anti)-chiral supermanifolds as

$$
\begin{gathered}
L_{b} \longrightarrow \tilde{L}_{b}^{(b)}=p_{\mu}(\tau) \dot{X}^{\mu(b)}(\tau, \bar{\theta})-\frac{1}{2} E^{(b)}(\tau, \bar{\theta})\left(p^{2}(\tau)-m^{2}\right)+b(\tau) \dot{E}^{(b)}(\tau, \bar{\theta}) \\
+\frac{1}{2} b^{2}(\tau)-i \dot{\bar{F}}^{(b)}(\tau, \bar{\theta}) \dot{F}^{(b)}(\tau, \bar{\theta}), \\
L_{b} \longrightarrow \tilde{L}_{b}^{(a b)}=p_{\mu}(\tau) \dot{X}^{\mu(a b)}(\tau, \theta)-\frac{1}{2} E^{(a b)}(\tau, \theta)\left(p^{2}(\tau)-m^{2}\right)+b(\tau) \dot{E}^{(a b)}(\tau, \theta) \\
+\frac{1}{2} b^{2}(\tau)-i \dot{\bar{F}}^{(a b)}(\tau, \theta) \dot{F}^{(a b)}(\tau, \theta),
\end{gathered}
$$

where we have taken into account the (anti-)BRST invariance (i.e. $s_{(a) b} p_{\mu}=0, s_{(a) b} b=0$ ) of the variables $p_{\mu}(\tau)$ and $b(\tau)$ so that $\tilde{B}^{(b)}(\tau, \bar{\theta})=b(\tau), P_{\mu}^{(b)}(\tau)=p_{\mu}(\tau), \tilde{B}^{(a b)}(\tau, \bar{\theta})=$ $b(\tau), P_{\mu}^{(b)}(\tau)=p_{\mu}(\tau)$ are the trivial expansions. It is elementary to check that

$$
\begin{array}{lll}
\frac{\partial}{\partial \bar{\theta}} \tilde{L}_{0}=\frac{d}{d \tau}\left[\frac{1}{2} c\left(p^{2}+m^{2}\right)+b \dot{c}\right] & \Longleftrightarrow & s_{b} L_{B}, \\
\frac{\partial}{\partial \theta} \tilde{L}_{0}=\frac{d}{d \tau}\left[\frac{1}{2} \bar{c}\left(p^{2}+m^{2}\right)+b \dot{\bar{c}}\right] & \Longleftrightarrow & s_{a b} L_{B},
\end{array}
$$

which shows the (anti-)BRST invariance of the action integral $S=\int d \tau L_{b}$ within the framework of (anti-)chiral supervariable approach to BRST formalism. In other words, we have captured the (anti-)BRST invariance (cf. Eq. (3)) of Lagrangian $L_{b}$ in the terminology of (anti-)chiral supervariable approach to BRST formalism in the above Eq. (C.11).

We would like to offer a comment here on the above equation (C.11). Precisely speaking, we should take the derivative w.r.t. $\tau$, on the r.h.s. of Eq. (C.11), as the partial derivative because $\tau$ is a part of the superspace coordinates $(\tau, \bar{\theta})$ and $(\tau, \theta)$ that characterize the appropriately chosen $(1,1)$-dimensional (anti-)chiral supermanifolds on which the supervariables are defined. It is the latter entities that have been taken into consideration for our discussion on the (anti-)chiral supervariable approach to BRST formalism.

\section{References}

[1] J. Thierry-Mieg, J. Math. Phys. 21, 2834 (1980) 
[2] M. Quiros, F. J. De Urries, J. Hoyos, M. L. Mazon, E. Rodrigues, J. Math. Phys. 22, 1767 (1981)

[3] R. Delbourgo, P. D. Jarvis, J. Phys. A: Math. Gen. 15, 611 (1981)

[4] L. Bonora, M. Tonin, Phys. Lett. B 98, 48 (1981)

[5] L. Bonora, P. Pasti, M. Tonin, Nuovo Cimento A 64, 307 (1981)

[6] L. Bonora, P. Pasti, M. Tonin, Annals of Physics 144, 15 (1982)

[7] L. Baulieu, J. Thierry-Mieg, Nucl. Phys. B 197, 477 (1982)

[8] L. Alvarez-Gaume, L. Baulieu, Nucl. Phys. B 212, 255 (1983)

[9] G. Curci, R. Ferrari, Phys. Lett. B 63, 91 (1976)

[10] L. Bonora, R. P. Malik, Phys. Lett. B 655, 75 (2007)

[11] L. Bonora, R. P. Malik, J.Phys. A 43, 375403 (2010)

[12] See, e.g., R. P. Malik, J. Phys. A: Math. Gen. 37, 5261 (2004)

[13] See, e.g., R. P. Malik, J. Phys. A: Math. Theor. 39, 10575 (2006)

[14] See, e.g., R. P. Malik, Eur. Phys. J. C 51, 169 (2007)

[15] See, e.g., R. P. Malik, Eur. Phys. J. C 60, 457 (2009)

[16] See, e.g., A. Shukla, S. Krishna, R. P. Malik

Advances in High Energy Physics 2015, 258536 (2015)

[17] N. Srinivas, T. Bhanja, R. P. Malik, arXiv: 1504.04237 [hep-th]

[18] T. Bhanja, N. Srinivas, R. P. Malik Advances in High Energy Physics 2016, 3673206 (2016)

[19] N. Srinivas, T. Bhanja, R. P. Malik, Advances in High Energy Physics 2017, 6138263 (2017)

[20] A. Shukla, N. Srinivas, R. P. Malik, Annals of Physics 394, 98 (2018)

[21] B. Chauhan, S. Kumar, R. P. Malik, Int. J. Mod. Phys. A 33, 1850026 (2018)

[22] S. Kumar, B. Chauhan, R. P. Malik, arXiv: 1712.05242 [hep-th]

[23] A. Shukla, S. Krishna, R. P. Malik, Eur. Phys. J. C 72, 2188 (2012)

[24] See, e.g., D. Nemschansky, C. Preitschopf, M. Weinstein, Annals of Physics 183, 226 (1988)

[25] R. P. Malik, Mod. Phys. Lett. A 20, 1767 (2005) 
[26] P. A. M. Dirac, Lectures on Quantum Mechanics, (Yeshiva University Press, New York, 1964)

[27] K. Sundermeyer, Constrained Dynamics: Lecture Notes in Physics Vol. 169, (Springer, New York, 1982)

[28] N. Nakanishi, I. Ojima, Covariant Operator Formalism of Gauge Theories and Quantum Gravity (World Scientific, Singapore, 1990)

[29] S. Krishna, A. Shukla, R. P. Malik, Int. J. Mod. Phys. A 29, 1450183 (2014)

[30] S. Krishna, R. P. Malik, Annals of Physics 355, 204 (2015)

[31] S. Krishna, R. P. Malik, Europhys. Lett. 109, 31001 (2015)

[32] S. Krishna, D. Shukla, R. P. Malik, Int. J. Mod. Phys. A 31, 1650113 (2016)

[33] R. P. Malik, etal., in preparation 